Original Research Paper

\title{
Analysis of Low Probability of Intercept Radar Signals Using the Reassignment Method
}

\author{
${ }^{1}$ Daniel L. Stevens and ${ }^{2}$ Stephanie A. Schuckers \\ ${ }^{1}$ Air Force Research Laboratory, Rome, NY 13441 United States \\ ${ }^{2}$ Department of Electrical and Computer Engineering, Clarkson University, Potsdam, NY 13699, United States
}

Article history

Received: 22-09-2014

Revised: 24-09-2014

Accepted: 13-02-2015

Corresponding Author:

Daniel L. Stevens

Air Force Research Laboratory,

Rome, NY 13441, United States

Email: daniel.stevens.7@us.af.mil

\begin{abstract}
Digital intercept receivers are currently moving towards classical time-frequency analysis techniques for analyzing low probability of intercept radar signals. Although these techniques are an improvement over existing Fourier-based techniques, they still suffer from a lack of readability, due to poor time-frequency localization and cross-term interference, which may lead to inaccurate detection and parameter extraction. In this study, the reassignment method, because of its ability to smooth out cross-term interference and improve timefrequency localization, is proposed as an improved signal analysis technique to address these deficiencies. Simulations are presented that compare time-frequency representations of the classical timefrequency techniques with those of the reassignment method. Four different low probability of intercept waveforms are analyzed (two frequency modulated continuous wave waveforms and two frequency shift keying waveforms). The following metrics are used for analysis evaluation: Percent detection, number of cross-term false positives, lowest signal-to-noise ratio for signal detection, processing time, percent error of: Carrier frequency, modulation bandwidth, modulation period, time-frequency localization ( $\mathrm{x}$ and $\mathrm{y}$ direction) and chirp rate. Experimental results demonstrate that the 'squeezing' and 'smoothing' qualities of the reassignment method improve readability over the classical time-frequency analysis techniques and consequently, provide more accurate signal detection and parameter extraction in all but one of the metrics categories.
\end{abstract}

Keywords: Electronic Countermeasures, Electronic Warfare, Radar Applications, Radar Detection

\section{Introduction}

Today, radar systems face serious threats from antiradiation systems (Faulconbridge, 2002), electronic attack (Gau, 2002) and other areas. In order to be able to perform their functions properly, they must be able to 'see without being seen' (Pace, 2009; Wiley, 2006). One class of radars, Low Probability of Intercept (LPI) radars address this need through very low peak power, wide bandwidth, high duty cycle and power management, making them difficult to be detected and characterized by intercept receivers.

An intercept receiver that intercepts LPI radar signals faces threats as well. It must be able to detect and characterize the signals from LPI radars for the purpose of providing timely information about threatening systems. In addition, LPI radar intercept receivers provide information about defensive systems, which is necessary to maintain a credible deterrent force to penetrate other's defenses. Knowledge provided by the intercept receiver provides insight for determining the details of the threat so that an effective response can be prepared (Wiley, 2006).

To make matters even more challenging, most intercept receivers currently in use are analog, which were designed to intercept 'older' (i.e., non-LPI) radar signals and which perform poorly when faced with LPI radar signals. Digital receivers, which are emerging, are seen as the eventual solution to the LPI challenge of the intercept receiver. 
Fourier analysis techniques using the Fast Fourier Transform (FFT) have been employed as the basic tool of the digital intercept receiver for detecting and extracting parameters of LPI radar signals and make up a majority of the digital intercept receiver techniques currently in the fleet (Pace, 2009). When a practical no stationary signal (such as an LPI radar signal) is processed, the Fourier transform, as implemented, cannot efficiently analyze and process the time-varying characteristics of the frequency spectrum of the signal (Xie et al., 2008; Stephens, 1996). The non-stationary nature of the received radar signal mandates the use of some form of timefrequency analysis for signal detection and parameter extraction (Milne and Pace, 2002).

Some of the more common classical time-frequency analysis techniques include the Wigner-Ville Distribution (WVD), Choi-Williams Distribution (CWD), spectrogram and scalogram. The WVD exhibits the highest signal energy concentration (Wiley, 2006), but has the worse cross-term interference, which can severely limit the readability of a time-frequency representation (Gulum, 2007; Stephens, 1996; Boashash, 2003). The CWD is a member of Cohen's class, which adds a smoothing kernel to help reduce cross-term interference (Boashash, 2003; Upperman, 2008). The CWD, as with all members of Cohen's class, is faced with a trade-off between cross-term reduction and timefrequency localization. The spectrogram is the magnitude squared of the short-time Fourier transform (Hlawatsch and Boudreaux-Bartels, 1992; Mitra, 2001). It has poorer time-frequency localization but less crossterm interference than either the WVD or CWD and its cross-terms are limited to regions where the signals overlap (Auger et al., 1996). The scalogram is the magnitude squared of the wavelet transform and can be used as a time-frequency distribution (Cohen, 2002; Galleani et al., 2006; Cirillo et al., 2008). Like the spectrogram, the scalogram has cross-terms that are limited to regions where the signals overlap (Auger et al., 1996; Hlawatsch and Boudreaux-Bartels, 1992). Currently, for digital intercept receivers, these classical time-frequency analysis techniques, which are the current state-of-the-art techniques in this arena, are primarily at the lab phase (Pace, 2009).

Though classical time-frequency analysis techniques, such as those described above, are a great improvement over Fourier analysis techniques, they suffer in general from poor time-frequency localization and cross-term interference, as described above. This may result in degraded readability of time-frequency representations, potentially leading to inaccurate LPI radar signal detection and parameter extraction metrics and as such, can lead to decisions based on inaccurate information.
A promising avenue for overcoming these deficiencies is the utilization of the reassignment method. The reassignment method, which can be applied to most energy distributions (Hippenstiel et al., 2000), has, in theory, a perfectly localized distribution for chirps, tones and impulses (Auger et al., 1996; Boashash, 2003), making it a good candidate for the analysis of certain LPI radar signals, such as the triangular modulated Frequency Modulated Continuous Wave (FMCW) (which can be viewed as back-to-back chirps) and the Frequency Shift Keying (FSK) (which can be viewed as tones).

In related work, (Hippenstiel et al., 2000) performed comparisons between the reassignment method and the WVD and spectrogram, but this was done predominantly for a single chirp signal, which presents no cross-term interference. It appears that there has been limited research done in the area of utilization of the reassignment method for the analysis of triangular modulated FMCW LPI radar signals and FSK LPI radar signals.

\section{Background}

The derivation of the reassigned spectrogram expression starts with the assumption that the spectrogram can be defined as a two-dimensional convolution of the WVD of the signal by the WVD of the analysis window, yielding Equation 1:

$$
S_{x}(t, f ; h)=\int_{-\infty}^{+\infty} \int_{x}(s, \xi) W_{h}(t-s, f-\xi) d s d \xi
$$

Where:

$$
\begin{aligned}
& t=\text { Time } \\
& f=\text { Frequency } \\
& h=\text { Window size } \\
& x(t)=\text { The analyzed signal } \\
& h(t)=\text { The analyzing window } \\
& W_{x}=\text { The Wigner-Ville distribution of the signal } \\
& W_{h}=\text { The Wigner-Ville distribution of the window }
\end{aligned}
$$

The distribution reduces the interference terms of the signal's WVD, but at the expense of time and frequency localization. However, a closer look at (1) shows that $W_{h}(t-s, f-\xi)$ delimits a time-frequency domain at the vicinity of the $(t, f)$ point, inside which a weighted average of the signal's WVD values is performed. The key point of the reassignment principle is that these values have no reason to be symmetrically distributed around $(t, f)$, which is the geometrical center of this domain. Therefore, their average should not be assigned at this point, but rather at the center of gravity of this domain, which is much more representative of the local energy distribution of 
the signal (Auger and Flandrin, 1994). Reasoning with a mechanical analogy, the local energy distribution $W_{h}(t-s, f-\xi) W_{x}(s, \xi)$ (as a function of $s$ and $\xi$ ) can be considered as a mass distribution and it is much more accurate to assign the total mass (i.e., the spectrogram value) to the center of gravity of the domain rather than to its geometrical center (Boashash, 2003).

The reassignment method moves each value of the spectrogram computed at any point $(t, f)$ to another point $(\hat{t}, \hat{f})$ which is the center of gravity of the signal energy distribution around $(t, f)(\mathrm{Li}$ and $\mathrm{Bi}, 2008)$, giving Equations 2 and 3:

$$
\begin{array}{r}
\hat{t}(x ; t, f)=\frac{\iint_{-\infty}^{+\infty} s W_{h}(t-s, f-\xi) W_{x}(s, \xi) d s d \xi}{\iint_{-\infty}^{+\infty} W_{h}(t-s, f-\xi) W_{x}(s, \xi) d s d \xi} \\
\hat{f}(x ; t, f)=\frac{\iint_{-\infty}^{+\infty} \xi W_{h}(t-s, f-\xi) W_{x}(s, \xi) d s d \xi}{\iint_{-\infty}^{+\infty} W_{h}(t-s, f-\xi) W_{x}(s, \xi) d s d \xi}
\end{array}
$$

This leads to the expression for the reassigned spectrogram, whose value at any point $\left(t^{\prime}, f^{\prime}\right)$ is the sum of all the spectrogram values reassigned to this point Equation 4:

$$
\begin{aligned}
& S_{x}^{(r)}\left(t^{\prime}, f^{\prime} ; h\right)=\iint_{-\infty}^{+\infty} S_{x}(t, f ; h) \delta\left(t^{\prime}-\hat{t}(x ; t, f)\right) \\
& \delta\left(f^{\prime}-\hat{f}(x ; t, f)\right) d t d f
\end{aligned}
$$

One of the most interesting properties of this new distribution is that it also uses the phase information of the STFT and not just its squared modulus as in the spectrogram. It uses this information from the phase spectrum to sharpen the amplitude estimates in time and frequency. This can be seen from the following expressions of the reassignment operators Equation 5 and 6:

$$
\begin{aligned}
& \hat{t}(x ; t, f)=-\frac{d \Phi_{x}(t, f ; h)}{d f} \\
& \hat{f}(x ; t, f)=f+\frac{d \Phi_{x}(t, f ; h)}{d t}
\end{aligned}
$$

where, $\Phi_{x}(t, f ; h)$ is the phase of the STFT of $x$ : $\Phi_{x}(t, f ; h)$ $=\arg \left(F_{x}(t, f ; h)\right)$. However, these expressions $((5)$ and (6)) do not lead to an efficient implementation and have to be replaced by (7) (local group delay) and (8) (local instantaneous frequency), giving Equation 7 and 8:

$$
\begin{aligned}
& \hat{t}(x ; t, f)=t-\mathfrak{R}\left\{\frac{F_{x}\left(t, f ; T_{h}\right) F_{x}^{*}(t, f ; h)}{\left|F_{x(t, f ; h)}\right|^{2}}\right\} \\
& \hat{f}(x ; t, f)=f-\mathfrak{I}\left\{\frac{F_{x}\left(t, f ; D_{h}\right) F_{x}^{*}(t, f ; h)}{\left|F_{x(t, f ; h)}\right|^{2}}\right\}
\end{aligned}
$$

where, $T_{h}(t)=t \times h(t)$ and $D_{h}(\mathrm{t})=\frac{d h}{d t}(t)$. This leads to an efficient implementation for the reassigned spectrogram without explicitly computing the partial derivatives of phase. The reassigned spectrogram may thus be computed by using 3 STFTs, each having a different window (the window function $h$; the same window with a weighted time ramp $t * h$; the derivative of the window function $h$ with respect to time $(d h / d t)$, (also known as the frequency-weighted window). Reassigned spectrograms are therefore very easy to implement and do not require a drastic increase in computational complexity.

Since time-frequency reassignment is not a bilinear operation, it does not permit a stable reconstruction of the signal. In addition, once the phase information has been used to reassign the amplitude coefficients, it is no longer available for use in reconstruction. This is perhaps why the reassignment method has received limited attention from engineers and why its greatest potential may be where reconstruction is not necessary, that is, where signal analysis is an end unto itself (Flandrin et al., 2003). The reassignment principle for the spectrogram allows for a straight-forward extension of its use to other distributions as well (Hippenstiel et al., 2000). If we consider the general expression of a distribution of the Cohen's class as a two-dimensional convolution of the WVD Equation 9:

$$
C_{x}(t, f ; \Pi)=\int_{-\infty}^{+\infty} \Pi(t-s, f-\xi) W_{x}(s, \xi) d s d \xi
$$

Then replacing the particular smoothing kernel $W_{h}(u, \xi)$ by an arbitrary kernel $\Pi(s, \xi)$ simply defines the reassignment of any member of Cohen's class, yielding Equation 10 to 12 :

$$
\begin{gathered}
\hat{t}(x ; t, f)=\frac{\iint_{-\infty}^{+\infty} s \Pi(t-s, f-\xi) W_{x}(s, \xi) d s d \xi}{\iint_{-\infty}^{+\infty} \Pi(t-s, f-\xi) W_{x}(s, \xi) d s d \xi} \\
\hat{f}(x ; t, f)=\frac{\iint_{-\infty}^{+\infty} \xi \Pi(t-s, f-\xi) W_{x}(s, \xi) d s d \xi}{\iint_{-\infty}^{+\infty} \Pi(t-s, f-\xi) W_{x}(s, \xi) d s d \xi}
\end{gathered}
$$




$$
\begin{aligned}
& C_{x}^{(r)}\left(t^{\prime}, f^{\prime} ; \Pi\right)=\iint_{-\infty}^{+\infty} C_{x}(t, f ; \Pi) \delta\left(t^{\prime}-\hat{t}(x ; t, f)\right) \\
& \delta\left(f^{\prime}-\hat{f}(x ; t, f)\right) d t d f
\end{aligned}
$$

The resulting reassigned distributions efficiently combine a reduction of the interference terms provided by a well adapted smoothing kernel and an increased localization of the signal components achieved by the reassignment. In addition, the reassignment operators $\hat{t}(x ; t, f)$ and $\hat{f}(x ; t, f)$ are almost as easy to compute as for the spectrogram (Auger and Flandrin, 1995).

Similarly, the reassignment method can also be applied to the time-scale energy distributions. Starting from the general expression Equation 13:

$$
\Omega_{x}(t, a ; \Pi)=\iint_{-\infty}^{+\infty} \Pi\left(s / a, f_{0}-a \xi\right) W_{x}(t-s, \xi) d s d \xi
$$

It can be seen that the representation value at any point $\left(t, a=f_{0} / f\right)$ is the average of the weighted WVD values on the points $(t-s, \xi)$ located in a domain centered on $(t, f)$ and bounded by the essential support of $\Pi$. In order to avoid the resultant signal components broadening while preserving the cross-terms attenuation, it seems once again appropriate to assign this average to the center of gravity of these energy measures, whose coordinates are Equation 14 and 15:

$$
\hat{t}(x ; t, f)=t-\frac{\iint_{-\infty}^{+\infty} s \Pi\left(s / a, f_{0}-a \xi\right) W_{x}(t-s, \xi) d s d \xi}{\iint_{-\infty}^{+\infty} \Pi\left(s / a, f_{0}-a \xi\right) W_{x}(t-s, \xi) d s d \xi}
$$

$$
\begin{aligned}
& \hat{f}(x ; t, f)=\frac{f_{0}}{\hat{a}(x ; t, f)} \\
& =\frac{\iint_{-\infty}^{+\infty} \xi \Pi\left(s / a, f_{0}-a \xi\right) W_{x}(t-s, \xi) d s d \xi}{\iint_{-\infty}^{+\infty} \Pi\left(s / a, f_{0}-a \xi\right) W_{x}(t-s, \xi) d s d \xi}
\end{aligned}
$$

Rather than assign the average to the point $\left(t, a=f_{0} / f\right)$ where it is computed. The value of the resulting modified time-scale representation on any point $\left(t^{\prime}, a^{\prime}\right)$ is then the sum of all the representation values moved to this point and is known as the reassigned scalogram Equation 16:

$$
\begin{aligned}
& \Omega_{x}^{(r)}\left(t^{\prime}, a^{\prime} ; \Pi\right)=\iint_{-\infty}^{+\infty} a^{\prime 2} \Omega_{x}(t, a ; \Pi) \delta\left(t^{\prime}-\hat{t}(x ; t, a)\right) \\
& \delta\left(a^{\prime}-\hat{a}(x ; t, a)\right) d t \frac{d a}{a^{2}}
\end{aligned}
$$

A potential drawback of the reassignment method is that there is a chance it could generate spurious artifacts due to the reassignment process randomly clustering noise (vice signals). Because of this, its performance may suffer in low Signal-to-Noise Ratio (SNR) scenarios (Boashash, 2003).

The reassignment method can be viewed as helping to build a more readable time-frequency representation. The first step in the process is to reduce (smooth) the cross-term interference. An unfortunate side-effect of this smoothing is that the signal components become smeared. The second step in the process is then to refocus (squeeze) the components which were smeared during the smoothing process (Auger et al., 1996; Boashash, 2003). This 'smoothing' and 'squeezing' helps to create a more readable time-frequency representation.

In this study, the reassignment method is evaluated as a technique for improving the readability of the classical time-frequency analysis representations by improving time-frequency localization and reducing cross-term interference. This approach is assessed using 2 triangular modulated FMCW LPI radar signals and 2 FSK LPI radar signals (4-component and 8component). Metrics designed include percent error of: Carrier frequency, modulation bandwidth, modulation period, time-frequency localization and chirp rate. Additional metrics include: Percent detection, number of cross-term false positives, lowest SNR for signal detection and processing time.

The rest of this paper is organized as follows: Description of the proposed methodology is presented in the next section, followed by a section on the experimental results comparing the reassignment method and classical time-frequency analysis techniques. The last two sections of this paper consist of discussion and conclusions.

\section{Methodology}

The methodologies detailed in this paper describe the processes involved in obtaining and comparing metrics between the classical time-frequency analysis techniques and the reassignment method for the detection and parameter extraction of LPI radar signals.

The tools used for this testing were: Matrix Laboratory (MATLAB) (version 7.7), Signal Processing Toolbox (version 6.10), Wavelet Toolbox (version 4.3), Image Processing Toolbox (version 6.2), TimeFrequency Toolbox (version 1.0) (http://tftb.nongnu.org/). All the testing was accomplished on a desktop computer (Hewlett-Packard (HP) Compaq, $2.5 \mathrm{GHz}$ processor, Advanced Micro Devices (AMD) Athlon 64X2 Dual Core Processor 4800+, 2.00 GB Random Access Memory (RAM), 32 Bit Operating System). 
Testing was performed for 4 different waveforms (2 triangular modulated FMCWs and 2 FSKs-which are typical LPI waveforms), each waveform representing a different task (Task 1 through Task 4). For each waveform, parameters were chosen for academic validation of signal processing techniques. Due to computer processing limitations they were not meant to represent real-world values. The number of samples for each test was chosen to be either 256 or 512 , which seemed to be the optimum size for the desktop computer. Testing was performed at three different SNR levels: $10 \mathrm{~dB}, 0 \mathrm{~dB}$ and low SNR (the lowest SNR at which the signal could be detected). The noise added was white Gaussian noise, which best reflected the thermal noise present in the Intermediate Frequency (IF) section of an intercept receiver (Pace, 2009). Kaiser windowing was used, where windowing was applicable. 25 runs were performed for each test, for statistical purposes. The plots included in this study were done at a threshold of $5 \%$ of the maximum intensity and were linear scale (not $\mathrm{dB}$ ) of analytic (complex) signals; the color bar represents intensity. The signal processing tools used for each task were:

- Classical time-frequency analysis techniques: Spectrogram, scalogram, WVD, CWD

- Reassignment method: Reassigned spectrogram, reassigned scalogram, reassigned smoothed pseudo Wigner Ville distribution (RSPWVD)

Task 1 consisted of analyzing a triangular modulated FMCW signal (most prevalent LPI radar waveform (Liang et al., 2009)). The parameters were selected as: Sampling frequency $=4 \mathrm{KHz}$; carrier frequency $=1$ $\mathrm{KHz}$; modulation bandwidth $=500 \mathrm{~Hz}$; modulation period $=0.02 \mathrm{~s}$. Task 2 was similar to Task 1, but with different parameters: Sampling frequency $=6 \mathrm{KHz}$; carrier frequency $=1.5 \mathrm{KHz}$; modulation bandwidth $=$ $2.4 \mathrm{KHz}$; modulation period $=0.15 \mathrm{~s}$. The different parameters were chosen to see how the different shapes/heights of the triangles of the triangular modulated FMCW would affect the cross-term interference and the metrics.

Task 3 consisted of analyzing an FSK (prevalent in the LPI arena (Anjaneyulu et al., 2009)) 4-component signal whose parameters were selected as: Sampling frequency $=5 \mathrm{KHz}$; carrier frequencies $=1,1.75,0.75$, $1.25 \mathrm{KHz}$; modulation bandwidth $=1 \mathrm{KHz}$; modulation period $=0.025 \mathrm{~s}$.

Task 4 was similar to Task 3, but for an FSK 8component signal whose parameters were: Sampling frequency $=5 \mathrm{KHz}$; carrier frequencies $=1.5,1,1.25$, $1.5,1.75,1.25,0.75,1 \mathrm{KHz} ;$ modulation bandwidth $=1$ $\mathrm{KHz}$; modulation period $=0.0125 \mathrm{~s}$. The different number of components and different parameters between Task 3 and Task 4 were chosen to see how the different number/lengths of FSK components would affect the cross-term interference and the metrics.

Because of computational complexity, the WVD tests for 512 samples, $\mathrm{SNR}=0 \mathrm{~dB}$ and 512 samples, $\mathrm{SNR}=$ low SNR were not able to be performed for any of the 4 waveforms. It was noted that a single run was still processing after more than $8 \mathrm{~h}$. The WVD is known to be very computationally complex (Milne and Pace, 2002). After each particular run of each test, metrics were extracted from the time-frequency representation.

The metrics extracted were as follows:

- Processing time: Time required for plot to be displayed

- Percent detection: Percent of time signal was detected-signal was declared a detection if any portion of each of the signal components (4 chirp components for triangular modulated FMCW and 4 or 8 signal components for FSK) exceeded a set threshold (a certain percentage of the maximum intensity of the time-frequency representation)

Threshold percentages were determined based on visual detections of low SNR signals (lowest SNR at which the signal could be visually detected in the timefrequency representation) (Fig. 1).

Based on this analysis of visually detected low SNR plots for each signal processing tool, thresholds were assigned as follows: CWD (70\%); spectrogram (60\%); scalogram, reassigned spectrogram, reassigned scalogram, RSPWVD, WVD (4-component FSK) (50\%); WVD (triangular modulated FMCW) (35\%); WVD (8-component FSK) (20\%).

For percent detection determination, these threshold values were then included in the timefrequency plot algorithms so that the thresholds could be applied automatically during the plotting process. From the threshold plot, the signal was declared a detection if any portion of each of the signal components was visible (Fig. 2):

- Cross-Term False Positives (XFPs): The number of cross-terms that were wrongly declared as signal detections. For the time-frequency representation, the XFP detection criteria is the same as the timefrequency signal detection criteria listed in the percent detection section above

- Carrier frequency: The frequency corresponding to the maximum intensity of the time-frequency representation (there are multiple carrier frequencies for FSK waveforms)

- Modulation bandwidth: Distance from highest frequency value of signal (at a threshold of $20 \%$ maximum intensity) to lowest frequency value of signal (at same threshold) in Y-direction (frequency) 
CWD FSK 4-comp Fs $=5 \mathrm{KHz}$ modbw $=1 \mathrm{KHz}$ modper $=.025 \mathrm{~s}$ \# samples $=512 \mathrm{SNR}=-2 \mathrm{~dB}$

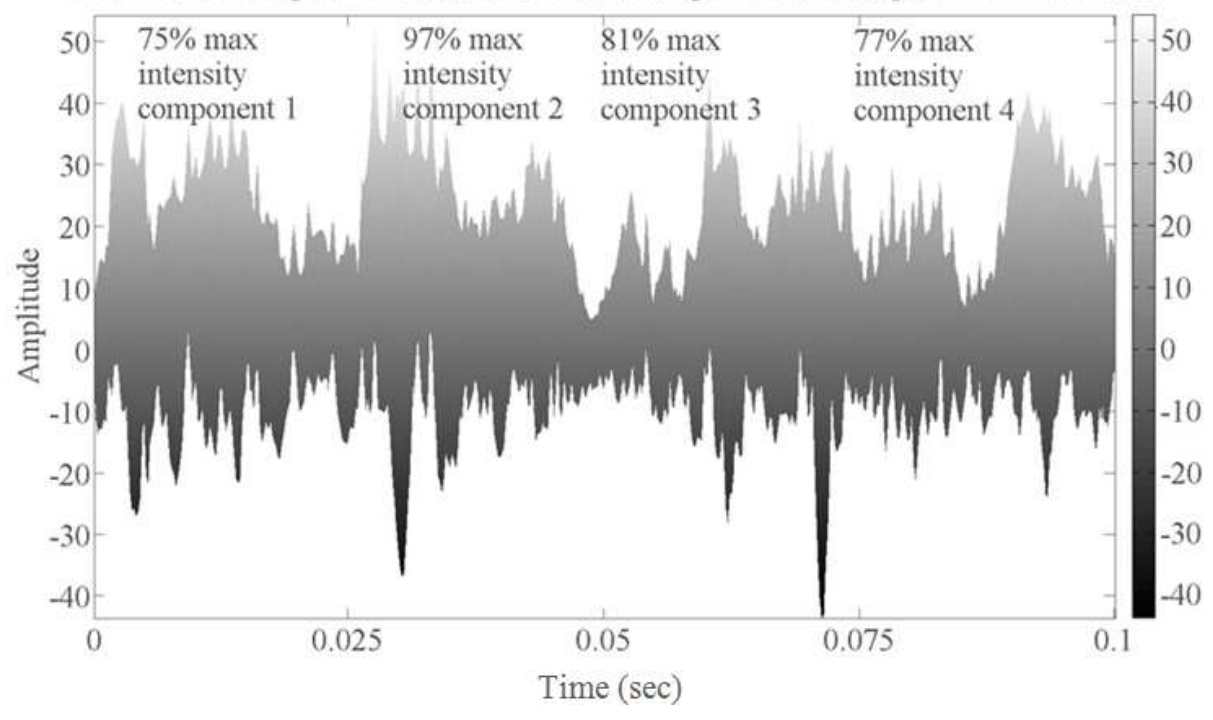

Fig. 1. Threshold percentage determination. This plot is an amplitude Vs. time (x-z view) of CWD of FSK 4-component signal (512 samples, $\mathrm{SNR}=-2 \mathrm{~dB}$ (the lowest SNR at which this signal could be visually detected)). For visually detected low SNR plots (like this one), the percent of maximum intensity for the peak z-value of each of the signal components was noted (here 75 , $97,81,77 \%$ ) and the lowest of these 4 values was recorded (75\%). Ten test runs were performed for each signal processing tool, for each of the 4 waveforms. The average of these recorded low values was determined and then assigned as the threshold for that particular signal processing tool. Note-the threshold for CWD was determined to be $70 \%$

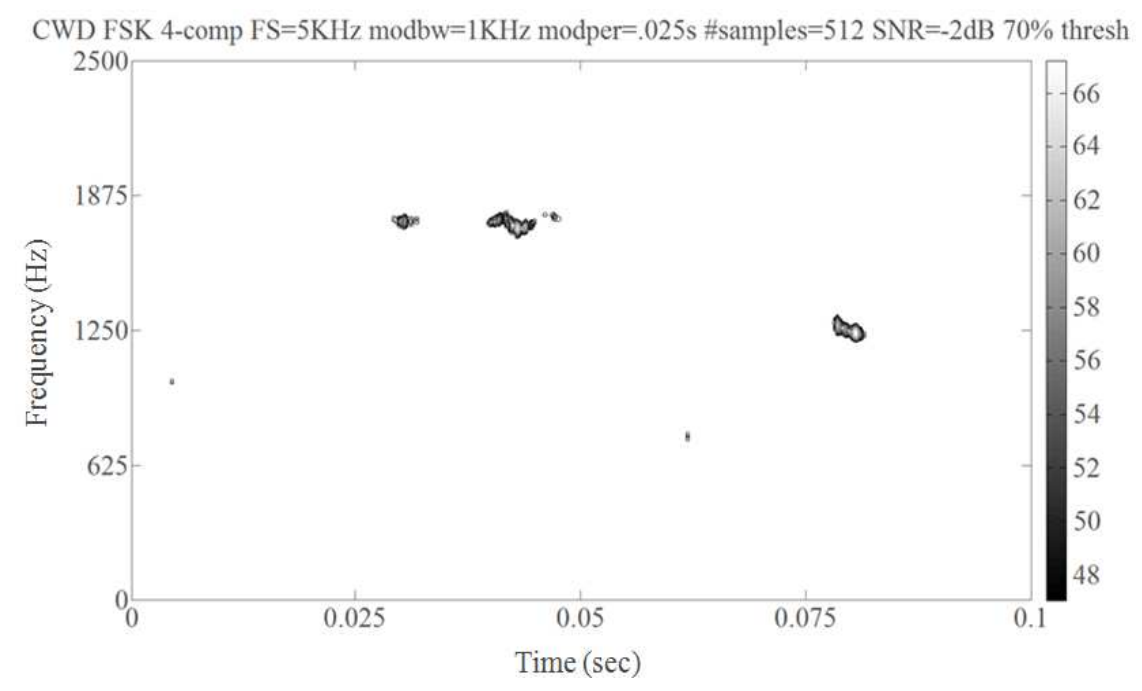

Fig. 2. Percent detection. Time Vs. Frequency view with threshold value automatically set to $70 \%$ (for CWD). From this $70 \%$ threshold plot, the signal was declared a detection because at least a portion of each of the 4 FSK signal components was visible

The threshold percentage was determined based on manual measurements of the modulation bandwidth of the signal in the time-frequency representation. This was accomplished for ten test runs for each signal processing tool, for each of the 4 waveforms. During each manual measurement, the maximum intensity of the two measuring points was recorded. The average of the maximum intensity values for these test runs was $20 \%$. This was adopted as the automatic threshold value and is representative of what is obtained when performing manual measurements.

For modulation bandwidth determination, the $20 \%$ threshold value was included in the time-frequency plot algorithms so that the threshold could be applied automatically during the plotting process. From the threshold plot, the modulation bandwidth was manually measured (Fig. 3 (for triangular modulated FMCW (solid arrows)) and Fig. 4 (for FSK (solid arrows)). 


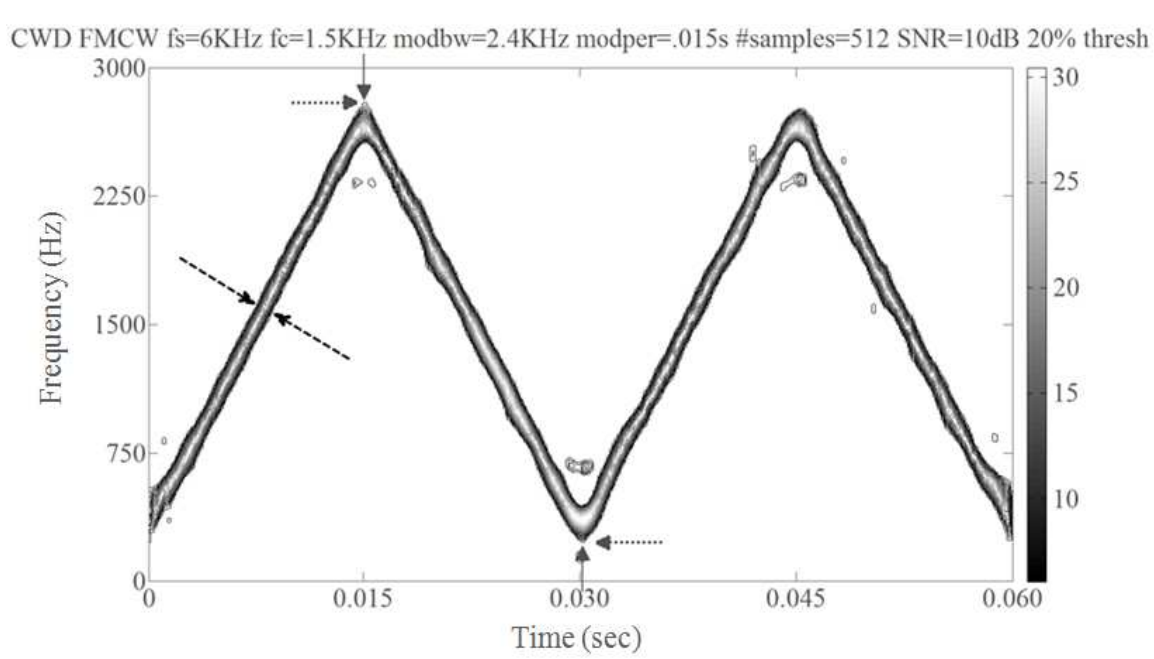

Fig. 3. Modulation bandwidth, modulation period and time-frequency localization determination (for triangular modulated FMCW waveform). CWD of a triangular modulated FMCW (512 samples, SNR $=10 \mathrm{~dB}$ ) with threshold value automatically set to $20 \%$. From this $20 \%$ threshold plot, the modulation bandwidth was measured manually from the highest frequency value of the signal (top solid arrow) to the lowest frequency value of the signal (bottom solid arrow) in the y-direction (frequency). The modulation period was measured manually from the highest frequency value of the signal (top dotted arrow) to the lowest frequency value of the signal (bottom dotted arrow) in the $\mathrm{x}$-direction (time). The time-frequency localization was a manual measurement of the 'thickness' of the signal component at the center of each of the 4 'legs' (distance between dashed arrows) and then the average of the 4 values was calculated and then converted to percent of entire $x$-axis and percent of entire $y$-axis

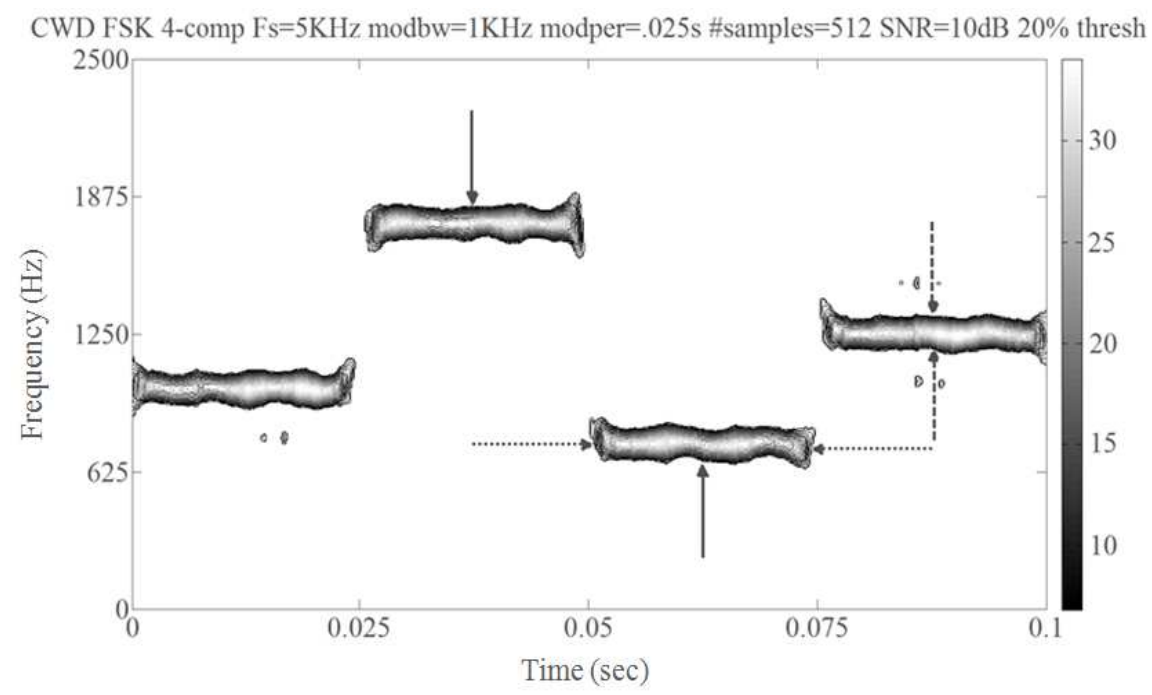

Fig. 4. Modulation bandwidth, modulation period and time-frequency localization determination (FSK waveform). CWD of a 4component FSK $(512$ samples, SNR $=10 \mathrm{~dB}$ ) with threshold value automatically set to $20 \%$. From this $20 \%$ threshold plot, the modulation bandwidth was measured manually from the highest frequency value of the signal (top solid arrow) to the lowest frequency value of the signal (bottom solid arrow) in the y-direction (frequency). The modulation period was measured manually as the width of each of the 4 FSK components in x-direction (time) (dotted arrows), then the average of the 4 signals was calculated. The time-frequency localization was a manual measurement of the 'thickness' of the signal component at the center of each of the 4 signal components (dashed arrows) and then the average of the 4 values was calculated and then converted to percent of entire $y$-axis

\section{Modulation Period}

For triangular modulated FMCW-distance from highest frequency value of signal (at a threshold of
$20 \%$ maximum intensity, which was determined using the same methodology as was used for the modulation bandwidth) to lowest frequency value of signal (at same threshold) in xdirection (time)-for FSK-width of 
FSK component (at a threshold of $20 \%$ maximum intensity) in xdirection (time). From Fig. 3 (triangular modulated FMCW signal), the modulation period is measured manually from the top dotted arrow to the bottom dotted arrow, but this time in the $\mathrm{x}$-direction (time). From Fig. 4 (FSK signal), the modulation period is the manual measurement of the width of each of the 4 signals in the $\mathrm{x}$-direction (time) (dotted arrows) and then the average of the 4 signals is calculated.

\section{Time-Frequency Localization}

A measure of the 'thickness' of a signal component (from one side of the signal component to the other) (at a threshold of $20 \%$ maximum intensity, which was determined using the same methodology as was used for the modulation bandwidth) on each side of the component-converted to \% of entire $\mathrm{x}$-Axis and $\%$ of entire y-Axis. From Fig. 3 (triangular modulated FMCW signal), the time-frequency localization is a manual measurement of the 'thickness' of the signal component at the center of each of the 4 'legs' (dashed arrows) and then the average of the 4 values is calculated. Average time and frequency 'thickness' values are then converted to: Percent of entire $\mathrm{x}$-axis and percent of entire y-axis. From Fig. 4 (FSK signal), the time-frequency localization is a manual measurement of the thickness of the center of each of the 4 signal components (y-direction only) (dashed arrows) and then the average of the 4 values is calculated. The average frequency 'thickness' is then converted to: Percent of the entire y-axis.

\section{Chirp Rate}

(modulation bandwidth)/(modulation period)-for Task 1 and Task 2 only.

\section{Lowest Detectable SNR}

The lowest SNR level at which at least a portion of each of the signal components exceeded the set threshold listed in the percent detection section above.

For lowest detectable SNR determination, these threshold values were included in the time-frequency plot algorithms so that the thresholds could be applied automatically during the plotting process. From the threshold plot, the signal was declared a detection if any portion of each of the signal components was visible. The lowest SNR level for which the signal was declared a detection is the lowest detectable SNR (Fig. 5).

Automatically applying a threshold value to the timefrequency plot algorithms for the determination of percent detection, modulation bandwidth, modulation period, time-frequency localization and lowest detectable SNR can be seen as a first step towards the future work of automating the metrics extraction process.

The data from all 25 runs for each test was used to produce the mean, standard deviation, variance, actual, error and percent error for each of the metrics listed above.

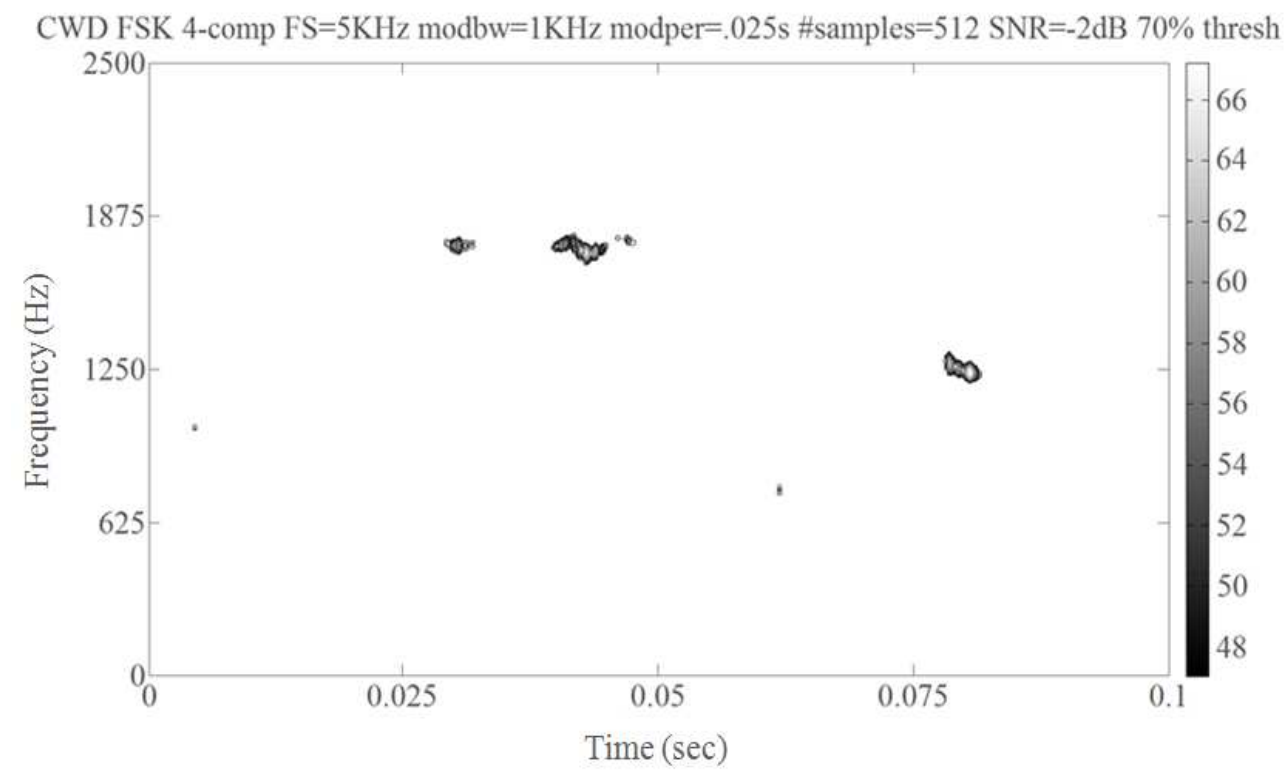

Fig. 5. Lowest detectable SNR. CWD of 4-component FSK (512 samples, SNR = -2 dB) (same as Fig. 2.) with threshold value automatically set to $70 \%$. From this threshold plot, the signal was declared a detection because at least a portion of each of the 4 FSK signal components was visible. For this case, any lower SNR would have been a non-detect 
The metrics from the classical time-frequency analysis techniques were then compared to the metrics from the reassignment method. By and large, the reassignment method outperformed the classical timefrequency analysis techniques, as will be shown in the results section.

\section{Results}

Some of the graphical and statistical results of the testing are presented in this section.

Table 1 represents the overall test metrics (signal processing tool viewpoint) average of all the signals for the 4 time-frequency analysis techniques and the 3 reassignment methods used in this testing.

From Table 1, the WVD has much better timefrequency localization percent error (x: 0.63\%/y: $1.45 \%$ ) than its classical time-frequency analysis techniques counterparts, the CWD (x: 1.88\%/y: $4.83 \%$ ), the spectrogram (x: $2.88 \% / y: 7.32 \%$ ) and the scalogram (x: $4.4 \% / y: 8.85 \%)$ and only slightly better time-frequency localization percent error than the reassigned spectrogram (x: $1.18 \% / y: 2.79 \%$ ) and the reassigned scalogram (x: $1.46 \% / y: 1.83 \%)$. The RSPWVD (x: $0.66 \% / y: 1.18 \%$ ) is nearly identical to the WVD in the $\mathrm{x}$-direction and slightly outperforms the WVD in the $y$-direction. Figure 6 shows that the reassigned spectrogram gives a more concentrated time-frequency localization than the spectrogram and that the reassigned scalogram gives a more concentrated time-frequency localization for each signal component than does the scalogram.

Table 1 also shows that the WVD has a large number of cross-term false positives (25). Figure 7 shows WVD cross-term interference, along with the ability of the RSPWVD to reduce these cross-terms, making for a more readable presentation.
Additionally, Table 1 shows that the WVD has the slowest processing time (1064 s) of all the techniques and that the processing times of the other 3 classical time-frequency analysis techniques, the CWD (10.34 s), the spectrogram $(4.25 \mathrm{~s})$ and the scalogram $(4.95 \mathrm{~s})$ are all faster than each of the 3 reassignment methods; the reassigned spectrogram $(49.1 \quad \mathrm{~s})$, the reassigned scalogram (13.63 s) and the RSPWVD (33.97 s). Also, Table 1 shows that the WVD has the worst low SNR value $(-2 \mathrm{~dB})$. In addition, the combined average for the reassignment method outperforms the combined average for the classical time-frequency analysis techniques in the areas of percent detection (98.9 to $93.4 \%$ ) and low SNR $(-2.81$ to $-2.58 \mathrm{~dB})$.

Table 2 presents the overall test metrics (SNR viewpoint) for the testing performed in this study and shows that the percent error of the modulation bandwidth, time-frequency localization, chirp rate, percent detection and processing time in general tend to worsen with lowering SNR values, for both the classical time-frequency analysis techniques and the reassignment method. Figure 8 gives a visual representation of the readability degradation that accompanies a reduction in SNR. The XFP numbers in Table 2 are representative of the fact that, due to computational complexity, there was no WVD testing accomplished at lower than $10 \mathrm{~dB}$ (except for the 256 sample cases).

Table 3 presents the overall test metrics (Task 1, 2, 3 and 4 viewpoint) for the testing performed in this paper and shows that the percent error of the modulation bandwidth, time-frequency localization (y-direction) and chirp rate are lower for Task 2 (triangular modulated FMCW, modulation bandwidth $=2.4 \mathrm{KHz}$ ) than for Task 1 (triangular modulated FMCW, modulation bandwidth $=500 \mathrm{~Hz}$ ) for both the classical time-frequency analysis techniques and the reassignment method. Figure 9 gives visual insight into this, as will be elaborated upon in the discussion section.

Table 1. Signal Processing Tool viewpoint of the overall test metrics (average percent error) for the 4 classical time-frequency analysis techniques (WVD (wvd), CWD (cwd), spectrogram (spectro), scalogram (scalo)), along with their combined average (TF) and for the 3 reassignment methods (reassigned spectrogram (respect), reassigned scalogram (rescalo), RSPWVD (rspwvd)), along with their combined average (RM). The parameters (Params) extracted are listed in the left-hand column: Carrier frequency (fc), modulation bandwidth (modbw), modulation period (modper), time-frequency localization in the xdirection (tf loc-x), time-frequency localization in the y-direction (tf loc-y), chirp rate (cr), percent detection (\% det), number of cross-term false positives (\#XFP), lowest detectable SNR (low snr), processing time (proctime)

\begin{tabular}{llllllllll}
\hline Params & wvd & cwd & spectro & scalo & TF & respect & rescalo & rspwvd & RM \\
\hline fc & $1.61 \%$ & $3.38 \%$ & $4.36 \%$ & $5.14 \%$ & $4.02 \%$ & $2.31 \%$ & $4.88 \%$ & $3.76 \%$ & $4.15 \%$ \\
modbw & $5.3 \%$ & $12.95 \%$ & $20.23 \%$ & $25.6 \%$ & $20.97 \%$ & $6.51 \%$ & $5.83 \%$ & $4.91 \%$ & $4.59 \%$ \\
modper & $7.5 \%$ & $7.1 \%$ & $4.95 \%$ & $4.52 \%$ & $6.02 \%$ & $4.59 \%$ & $3.11 \%$ & $4.72 \%$ & $4.14 \%$ \\
tf loc-x & $0.63 \%$ & $1.88 \%$ & $2.88 \%$ & $4.4 \%$ & $3.28 \%$ & $1.18 \%$ & $1.46 \%$ & $0.66 \%$ & $1.0 \%$ \\
tf loc-y & $1.45 \%$ & $4.83 \%$ & $7.32 \%$ & $8.85 \%$ & $6.42 \%$ & $2.79 \%$ & $1.83 \%$ & $1.18 \%$ & $1.94 \%$ \\
cr & $5.29 \%$ & $11.49 \%$ & $16.25 \%$ & $28.5 \%$ & $12.09 \%$ & $3.84 \%$ & $4.94 \%$ & $4.54 \%$ & $4.89 \%$ \\
\% det & $94.6 \%$ & $92.5 \%$ & $96.4 \%$ & $90.4 \%$ & $93.4 \%$ & $100 \%$ & $98.8 \%$ & $98.0 \%$ & $98.9 \%$ \\
\# XFP & 25 & 0 & 0 & 0 & 5.25 & 0 & 0 & 0 & 0 \\
low snr & $-2 \mathrm{db}$ & $-2.4 \mathrm{db}$ & $-3 \mathrm{db}$ & $-2.8 \mathrm{db}$ & $-2.58 \mathrm{db}$ & $-2.6 \mathrm{db}$ & $-2.8 \mathrm{db}$ & $-3 \mathrm{db}$ & $-2.81 \mathrm{db}$ \\
proctime & $1064 \mathrm{~s}$ & $10.34 \mathrm{~s}$ & $4.25 \mathrm{~s}$ & $4.95 \mathrm{~s}$ & $271 \mathrm{~s}$ & $49.1 \mathrm{~s}$ & $13.63 \mathrm{~s}$ & $33.97 \mathrm{~s}$ & $32.2 \mathrm{~s}$ \\
\hline
\end{tabular}


Table 2. SNR viewpoint of overall test metrics (average percent error) for the classical Time-Frequency analysis techniques (TF) and for the Reassignment Method (RM) for SNR $=10 \mathrm{~dB}, 0 \mathrm{~dB}$ and lowest detectable SNR (low snr). The parameters (Params) extracted are listed in the left-hand column: Carrier frequency ( $\mathrm{fc}$ ), modulation bandwidth (modbw), modulation period (modper), time-frequency localization in the x-direction (tf loc-x), time-frequency localization in the y-direction (tf loc-y), chirp rate (cr), percent detection (\% det), number of cross-term false positives (\#XFP), processing time (proctime)

\begin{tabular}{lllllll}
\hline Params & TF 10dB & TF 0dB & TF low snr & RM 10dB & RM 0dB & RM low snr \\
\hline fc & $4.89 \%$ & $2.00 \%$ & $5.31 \%$ & $4.12 \%$ & $2.54 \%$ & $2.30 \%$ \\
modbw & $14.78 \%$ & $15.4 \%$ & $18.81 \%$ & $4.44 \%$ & $5.25 \%$ & $7.46 \%$ \\
modper & $5.0 \%$ & $3.93 \%$ & $6.52 \%$ & $4.63 \%$ & $4.25 \%$ & $3.58 \%$ \\
tf loc-x & $2.34 \%$ & $2.45 \%$ & $2.61 \%$ & $0.78 \%$ & $1.01 \%$ & $1.41 \%$ \\
tf loc-y & $4.87 \%$ & $5.47 \%$ & $6.64 \%$ & $1.25 \%$ & $2.08 \%$ & $2.61 \%$ \\
cr & $13.73 \%$ & $14.51 \%$ & $17.04 \%$ & $4.82 \%$ & $3.35 \%$ & $5.14 \%$ \\
\% det & $100 \%$ & $82.4 \%$ & N/A & $100 \%$ & $97.9 \%$ & N/A \\
\# XFP & 21 & 2 & $421 \mathrm{~s}$ & 0 & 0 & 0 \\
proctime & $289 \mathrm{~s}$ & $234 \mathrm{~s}$ & $21.0 \mathrm{~s}$ & $23.65 \mathrm{~s}$ & $52.11 \mathrm{~s}$ \\
\hline
\end{tabular}

Table 3. Task 1, 2, 3 and 4 viewpoint of overall test metrics (average percent error) for the classical Time-Frequency analysis techniques (TF) and for the Reassignment Method (RM). Task1 = triangular modulated FMCW signal (modulation bandwidth $=500 \mathrm{~Hz}$ ), Task2 = triangular modulated FMCW signal (modulation bandwidth $=2.4 \mathrm{KHz})$, Task $3=$ FSK $(4-$ component) signal, Task 4 = FSK (8-component) signal. The parameters (Params) extracted are listed in the left-hand column: Carrier frequency (fc), modulation bandwidth (modbw), modulation period (modper), time-frequency localization in the $\mathrm{x}$-direction (tf loc-x), time-frequency localization in the $\mathrm{y}$-direction (tf loc-y), chirp rate (cr), percent detection (\% det), number of cross-term false positives (\#XFP), lowest detectable SNR (low snr), processing time (proctime)

\begin{tabular}{|c|c|c|c|c|c|c|c|c|}
\hline Params & TFTask1 & TFTask2 & TFTask3 & TFTask4 & RMTask1 & RMTask2 & RMTask3 & RMTask4 \\
\hline fc & $4.02 \%$ & $8.15 \%$ & $0.65 \%$ & $0.40 \%$ & $4.15 \%$ & $4.48 \%$ & $0.45 \%$ & $0.37 \%$ \\
\hline modbw & $20.97 \%$ & $5.90 \%$ & $19.37 \%$ & $20.67 \%$ & $4.59 \%$ & $3.36 \%$ & $7.35 \%$ & $8.27 \%$ \\
\hline modper & $0.70 \%$ & $0.41 \%$ & $10.7 \%$ & $17.47 \%$ & $0.78 \%$ & $0.56 \%$ & $6.25 \%$ & $12.0 \%$ \\
\hline tf loc-x & $2.78 \%$ & $2.55 \%$ & $7.50 \%$ & $8.50 \%$ & $1.00 \%$ & $1.28 \%$ & $2.27 \%$ & $2.43 \%$ \\
\hline tf loc-y & $6.42 \%$ & $2.45 \%$ & $\mathrm{~N} / \mathrm{A}$ & $\mathrm{N} / \mathrm{A}$ & $1.94 \%$ & $0.92 \%$ & $\mathrm{~N} / \mathrm{A}$ & $\mathrm{N} / \mathrm{A}$ \\
\hline $\mathrm{cr}$ & $12.09 \%$ & $5.77 \%$ & $\mathrm{~N} / \mathrm{A}$ & $\mathrm{N} / \mathrm{A}$ & $4.89 \%$ & $2.86 \%$ & $\mathrm{~N} / \mathrm{A}$ & $\mathrm{N} / \mathrm{A}$ \\
\hline$\%$ det & $88 \%$ & $93.7 \%$ & $100 \%$ & $100 \%$ & $97.33 \%$ & $100 \%$ & $100 \%$ & $100.0 \%$ \\
\hline \# XFP & 8 & 2 & 6 & 9 & 0 & 0 & 0 & 0 \\
\hline low snr & $-2.8 \mathrm{db}$ & $-3.3 \mathrm{db}$ & $-2.67 \mathrm{db}$ & $-1.67 \mathrm{db}$ & $-2.67 \mathrm{db}$ & $-3.0 \mathrm{db}$ & $-3.33 \mathrm{db}$ & $-2.5 \mathrm{db}$ \\
\hline proctime & $145 \mathrm{~s}$ & $439 \mathrm{~s}$ & $143 \mathrm{~s}$ & $104 \mathrm{~s}$ & $31.1 \mathrm{~s}$ & $35.3 \mathrm{~s}$ & $33.6 \mathrm{~s}$ & $22.8 \mathrm{~s}$ \\
\hline
\end{tabular}

Table 3 also shows that the percent error of the carrier frequency for Tasks 3 and 4 (FSK signals) is much lower than for Tasks 1 and 2 (triangular modulated FMCW signals). Also, the percent error of the modulation period for Tasks 1 and 2 (triangular modulated FMCW signals) is much lower than for Tasks 3 and 4 (FSK signals). In addition, the lowest detectable SNR is lower for Task 3 (4-component FSK signals) than for Task 4 (8-component FSK signal).

\section{Discussion}

This section of the paper will elaborate on the results from the previous section.

From Table 1 (signal processing tool viewpoint of overall test metrics), the performance of each of the 7 signal processing analysis tools will be summarized, including strengths, weaknesses and generic scenarios in which a particular tool might be used.

\section{WVD}

The WVD had excellent time-frequency localization percent error $(0.63 / 1.45 \%)$, but had the worst cross-term interference (25 XFPs), processing time (1064 s) and low
SNR (-2 dB). Its excellent time-frequency localization can be attributed to the fact that the WVD exhibits the highest signal energy concentration in the time-frequency plane (Gulum, 2007; Pace, 2009) and is totally concentrated along the instantaneous frequency (Cirillo et al., 2006; Guanghua et al., 2008). The cross-term interference problem of the WVD, which is well-known (Gulum, 2007), makes it very difficult to see the actual signal (De Luigi and Moreau, 2002; Guanghua et al., 2008; Wong et al., 2009), reducing the readability of the time-frequency distribution. Fig. 7 clearly shows the cross-term interference problem with the WVD. The cross-terms produced a false positive (XFP) triangle in the middle of the two-triangle signal (Fig. 7a) and also produced 9 XFPs in the FSK 8component signal (Fig. 7c). Cross-terms are located halfway between signal components (De Luigi and Moreau, 2002; Wong et al., 2009). The long processing time is indicative of the fact that the WVD is known to be computationally intensive (Milne and Pace, 2002), due in part to its cross-term interference. Though the WVD is highly concentrated in time and frequency, it is also highly non-linear and non-local and is therefore very sensitive to noise (Auger and Flandrin, 1995; Flandrin et al., 2003), which accounts for its poor low SNR performance $(-2 \mathrm{~dB})$ 
(De Luigi and Moreau, 2002; Guanghua et al., 2008). The WVD might be a good tool to use if excellent timefrequency localization is a requirement, but readability, speed and low SNR environments are not an issue, such as in a scenario where off-line analysis is performed, without any time constraints. The readability issue can be alleviated if a single-component signal is used, which would eliminate the cross-term interference, but which is unrealistic for most LPI radar signals.

\section{$C W D$}

The CWD did not perform the best or the worst in any one category. Two of its strongest areas were timefrequency localization percent error $(1.88 / 4.83 \%)$, where it performed better than the spectrogram and the scalogram and processing time (10.34 s) where it performed better than the WVD, reassigned spectrogram, reassigned scalogram and RSPWVD. The better than average performance in both time-frequency localization percent error and processing time can be attributed to the fact that the CWD is a member of the Cohen's class of time-frequency distributions, which use a smoothing kernel to smooth out cross-term interference, but at the expense of time-frequency localization (Choi and Williams, 1989; Williams and Jeong, 1992). In this sense, the CWD is seen as a 'mid-point' between the WVD (good localization, poor cross-term interference) and the spectrogram (poor localization, good cross-term interference). This reduction in cross-term interference speeds up the processing time of the CWD. The fact that the CWD does not smooth out all of the cross-term interference allows for adequate localization. 'Middle-ofthe-road' would be the best way to describe the performance of the CWD. The CWD might be used in a scenario where above average localization is required (i.e., somewhere between the WVD and the spectrogram) and where a fairly short processing time is required. The goal of such a scenario would be to obtain above average signal metrics in a short amount of time.

\section{Spectrogram}

The spectrogram had the best processing time $(4.25 \mathrm{~s})$, best low SNR $(-3 \mathrm{~dB})$ (tied with RSPWVD) and the best percent detection $(96.4 \%$ ) (of the classical time-frequency analysis techniques), but suffered in time-frequency localization percent error (2.88/7.32\%)-only the scalogram (4.4/8.85\%) was worse(Auger et al., 1996; Cohen, 1995; Hlawatsch and Boudreaus-Bartels, 1992). Both the good and the poor metrics can be accounted for by the extreme reduction of cross-term interference that the spectrogram provides, accounting for the quick processing time, low SNR and percent detection, but at the expense of poor timefrequency localization percent error. The spectrogram might be used in a scenario where short processing time is required, in a fairly low SNR environment and where timefrequency localization is not an issue. Such a scenario might be a 'quick and dirty' check to see if a signal is present, without precise extraction of its parameters.

\section{Scalogram}

The scalogram had good processing time (4.95 s), good low SNR $(-2.8 \mathrm{~dB})$, but had the worst percent detection $(90.4 \%)$ and time-frequency localization percent error $(4.4 / 8.85 \%)$. The scalogram suppresses almost all cross-terms (Grishin and Janczak, 2007; Lari and Zakhor, 1992), accounting for its good processing time and good low SNR performance. Because of this cross-term reduction, it is surprising that the scalogram did not perform better in the area of percent detection. This could be due to its large timefrequency localization percent error, or due to the fact that a wavelet/scalogram performs better on signals that change rapidly in frequency over time, vice the triangular modulated FMCW and FSK signals used in this study. Like the spectrogram, the scalogram might be used in a scenario where short processing time is required, in a fairly low SNR environment and where time-frequency localization is not an issue, or, as mentioned previously, in a scenario that detects/analyzes signals that change rapidly in frequency over time.

\section{Reassignment Method}

The three reassignment methods performed fairly similarly to one another, so they will be lumped together, with any specific differences being brought out separately. The reassignment methods performed very well in the time-frequency localization percent error category, coming close to the performance attained by the WVD (as mentioned earlier, the WVD exhibits the highest signal energy concentration in the time-frequency plane (Gulum, 2007; Pace, 2009)) and even surpassing the WVD in one area-time-frequency localization percent error in the $y$-direction $(1.18 \%$ for the RSPWVD Vs. $1.45 \%$ for the WVD)). Figure 6 displays this 'squeezing' quality of the reassignment method (Boashash, 2003). This quality is due to the fact that the reassignment method reassigns the signal energy value from the center of the analysis window, to the center of gravity of the analysis window, giving a much more concentrated signal representation (Auger and Flandrin, 1994; Li and $\mathrm{Bi}$, 2008). This supports the earlier hypothesis that, since the reassignment method is, in theory, a perfectly localized distribution for chirps, tones and impulses (Auger et al., 1996), it will work well for the triangular modulated FMCW (which can be viewed as back-to-back chirps) and the FSK (which can be viewed as tones), which can be seen in Fig. 6. Another presupposition was that the reassignment method produces a more concentrated signal (Auger et al., 1996), therefore the time-frequency distribution becomes more readable and a more readable time-frequency distribution makes for more accurate signal detection and parameter extraction metrics. The metrics in Table 1 confirm this, showing that the 
reassignment method by-and-large had more accurate signal detection and parameter extraction metrics than the classical time-frequency analysis techniques in the areas of percent error of the modulation bandwidth (4.59 to $20.97 \%$ ), percent error of time-frequency localization (both $\mathrm{x}(1.0$ to $3.28 \%$ ) and $\mathrm{y}(1.94$ to $6.42 \%)$ direction), percent error of the chirp rate (4.89 to $12.09 \%)$, percent detection (98.9 to $93.4 \%$ ), number XFPs (0 to 5.25$)$ and processing time (32.2s to $271 \mathrm{~s})$. Figure 7 is a prime example of the ability of the reassignment method to smooth out cross-term interference (Boashash, 2003). This figure shows how the readability of the time-frequency representation can be drastically increased through the cross-term reduction provided by the reassignment method. It was proposed that this increase in readability due to cross-term interference smoothing would bear an improvement over the classical time-frequency analysis techniques in the detection and parameter extraction metrics, which, as noted, is seen to be the case in Table 1. The 3 reassignment methods had slightly slower processing times than the classical time-frequency analysis techniques (except the WVD). This is due to the reassignment process being slightly more computationally complex than the CWD, spectrogram and scalogram. As far as the differences between the 3 reassignment methods; the RSPWVD had the best time-frequency localization percent error $(\mathrm{x}=0.66 \%, \mathrm{y}=1.18 \%)$ and best low SNR $(-3$ $\mathrm{dB})$; the reassigned scalogram had the fastest processing time (13.63 s) and the reassigned spectrogram had the best percent detection $(100 \%)$. Since the spectrogram has the worst localization (and the best cross-term interference) of any of Cohen's class (which would include the smoothedpseudo WVD (SPWVD)), then since the SPWVD yields better localization of signal components than the spectrogram, it follows that the reassigned version of the SPWVD, the RSPWVD, would be more localized ( $\mathrm{x}=$ $0.66 \%, \mathrm{y}=1.18 \%)$ than the reassigned spectrogram $(\mathrm{x}=$ $1.18 \%, y=2.79 \%$ ) and, following the same logic, also less sensitive to noise $(-3 \mathrm{~dB})$ than the reassigned spectrogram ($2.6 \mathrm{~dB}$ ) (Flandrin et al., 2003). Any of the 3 reassignment methods might be used in a scenario where excellent timefrequency localization and low cross-term interference is required, perhaps due to a crucial need for very accurate extraction of metrics.

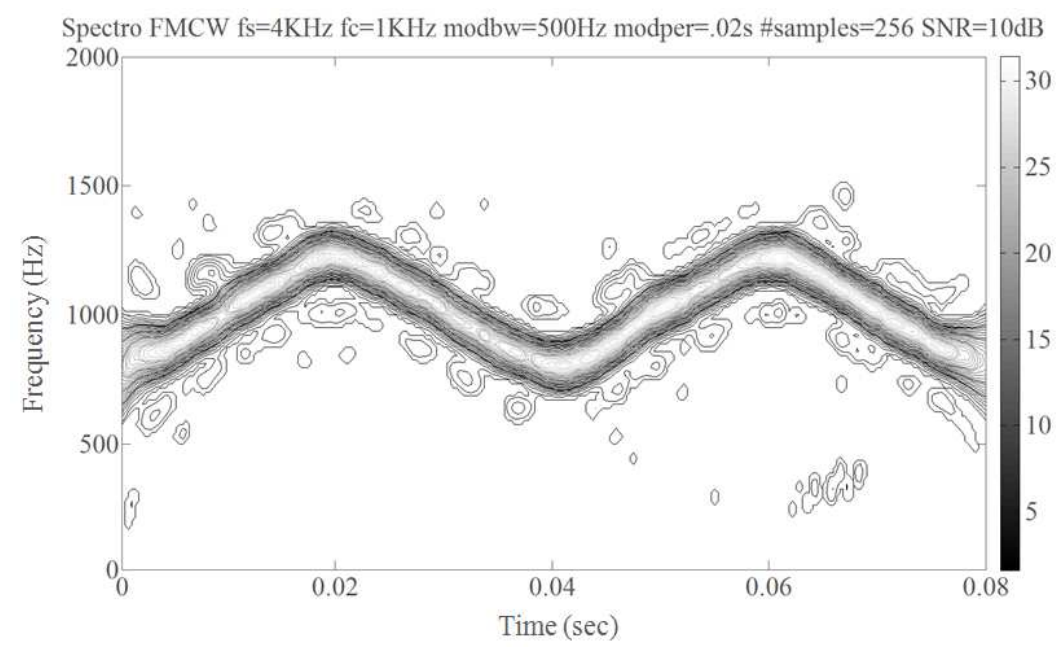

(a)

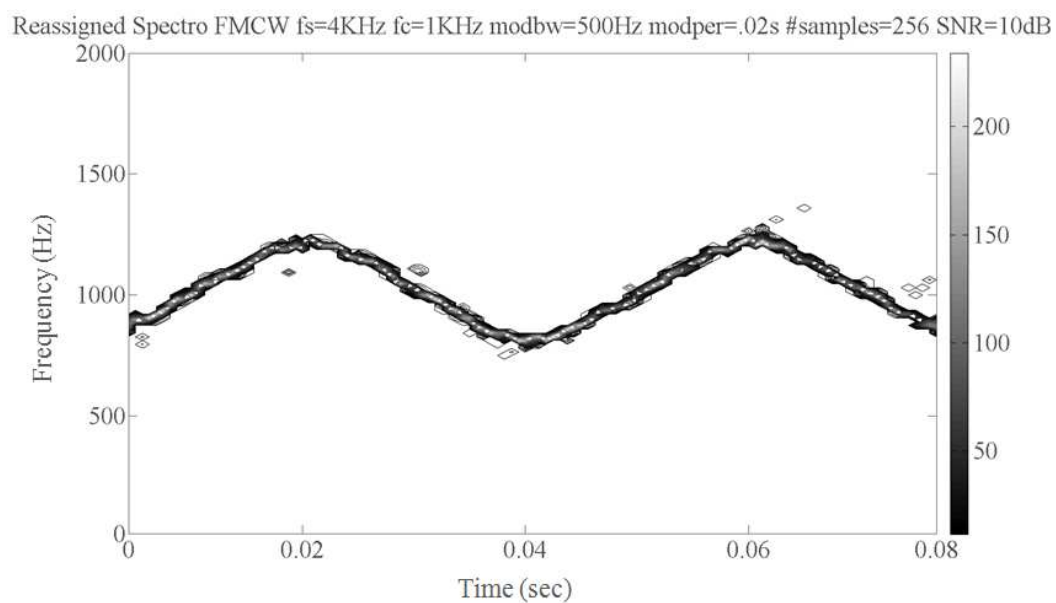

(b) 


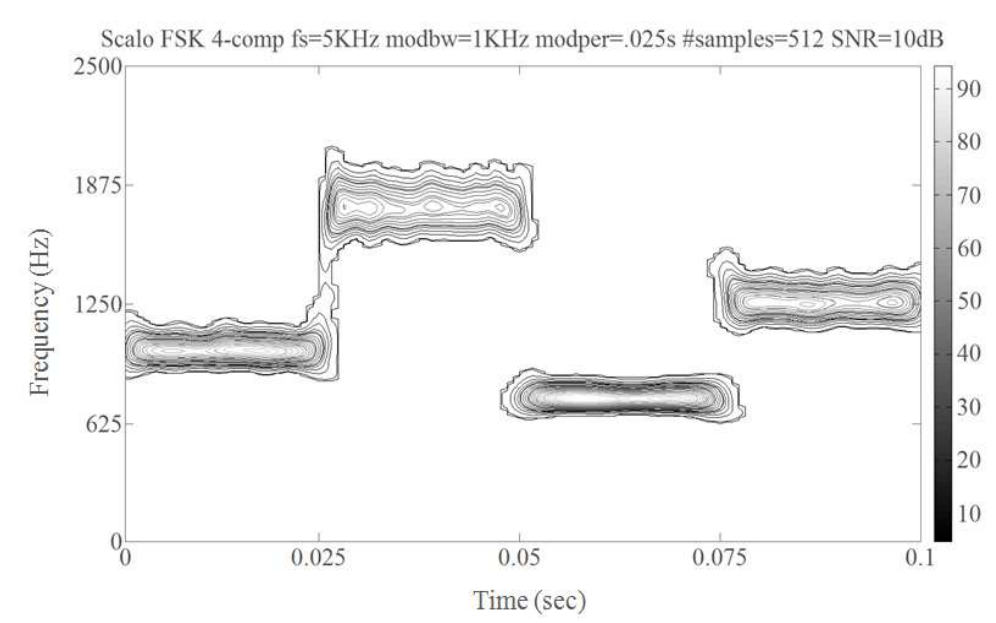

(c)

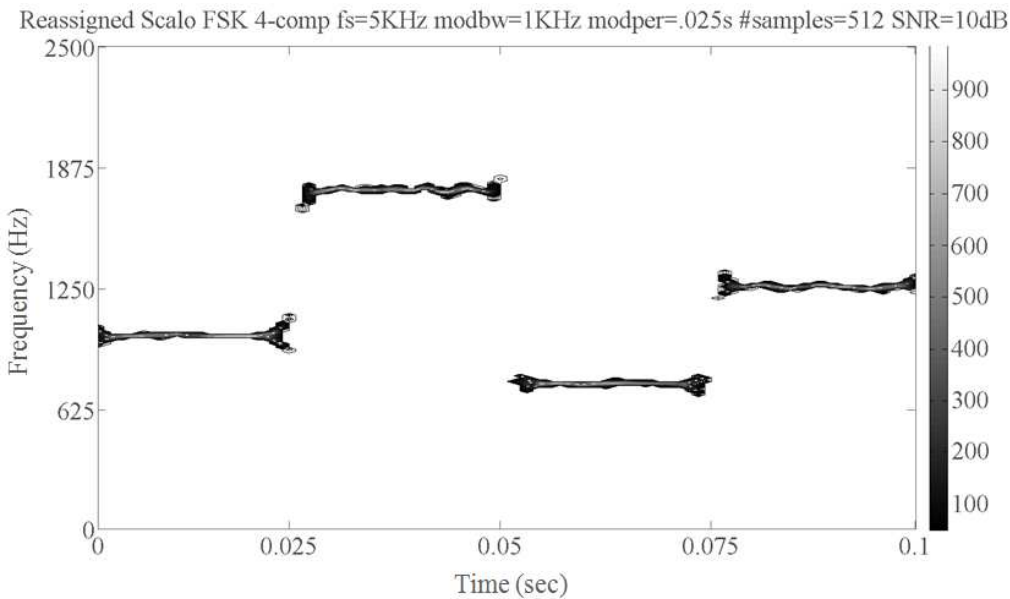

(d)

Fig. 6. Time-frequency localization comparison between the classical time-frequency analysis techniques and the reassignment method: (a) is the spectrogram and (b) is the reassigned spectrogram for a triangular modulated FMCW signal (256 samples, $\mathrm{SNR}=10 \mathrm{~dB})$; (c) is the scalogram and $(\mathrm{d})$ is the reassigned scalogram for a 4-component FSK signal (512 samples, $\mathrm{SNR}=$ $10 \mathrm{~dB}$ ). The reassignment method gives a much more concentrated time-frequency localization than does its classical timefrequency analysis counterpart

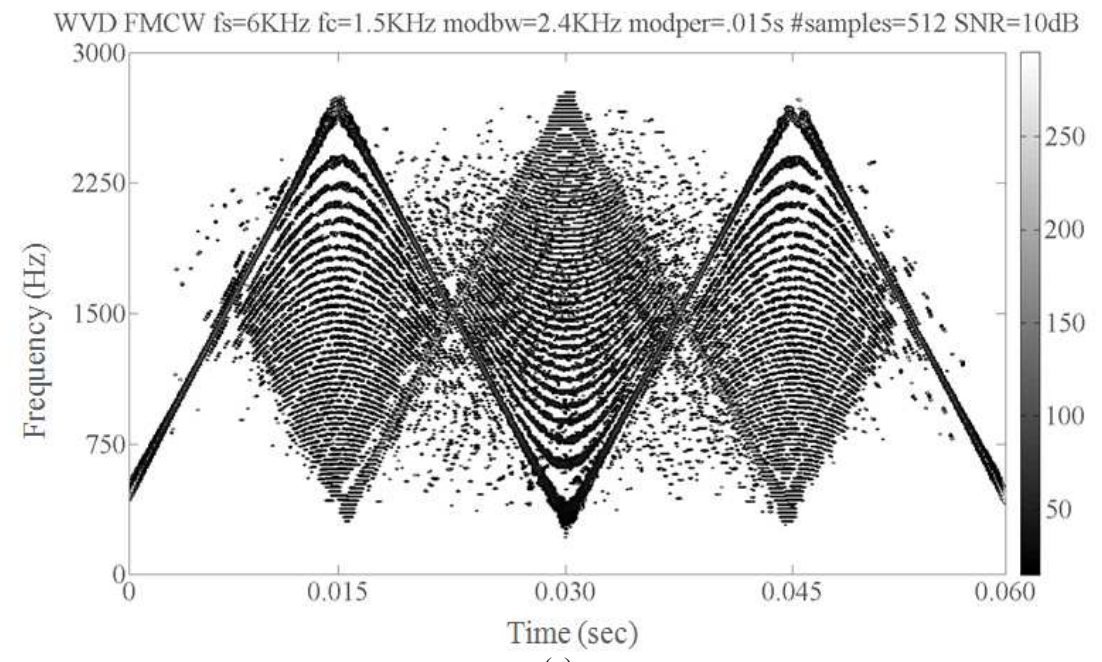

(a) 


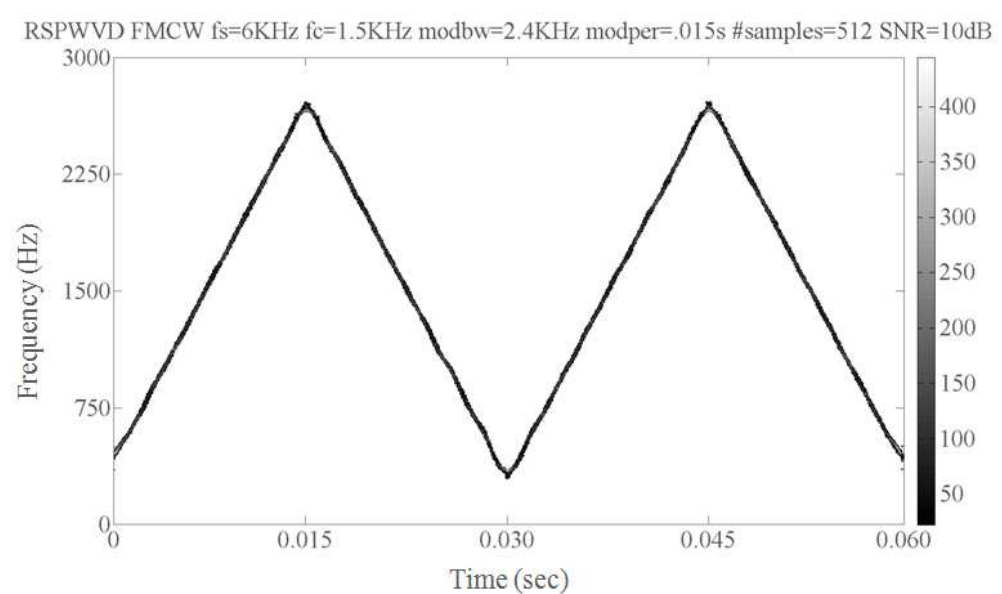

(b)

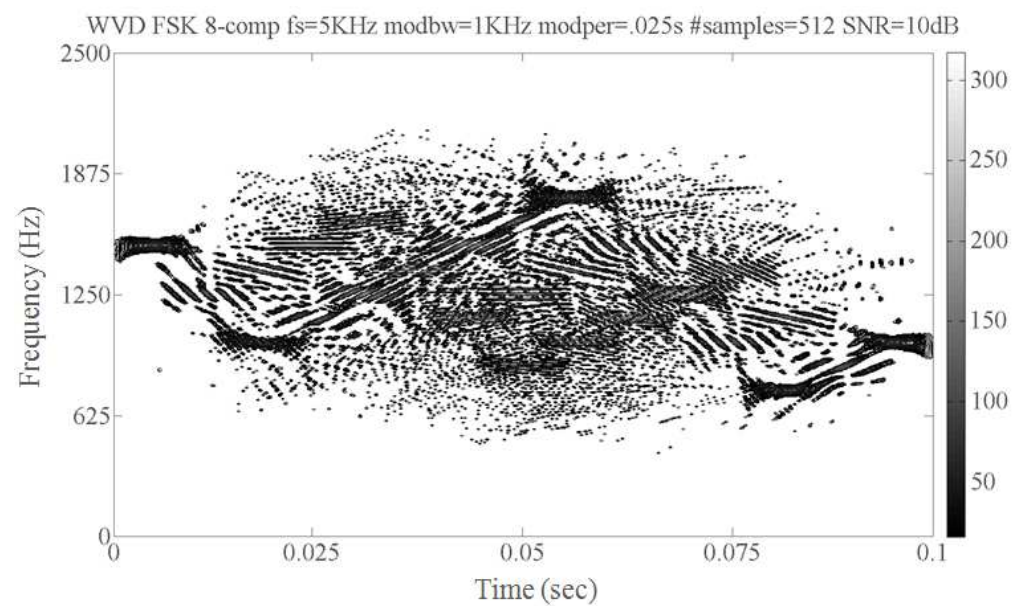

(c)

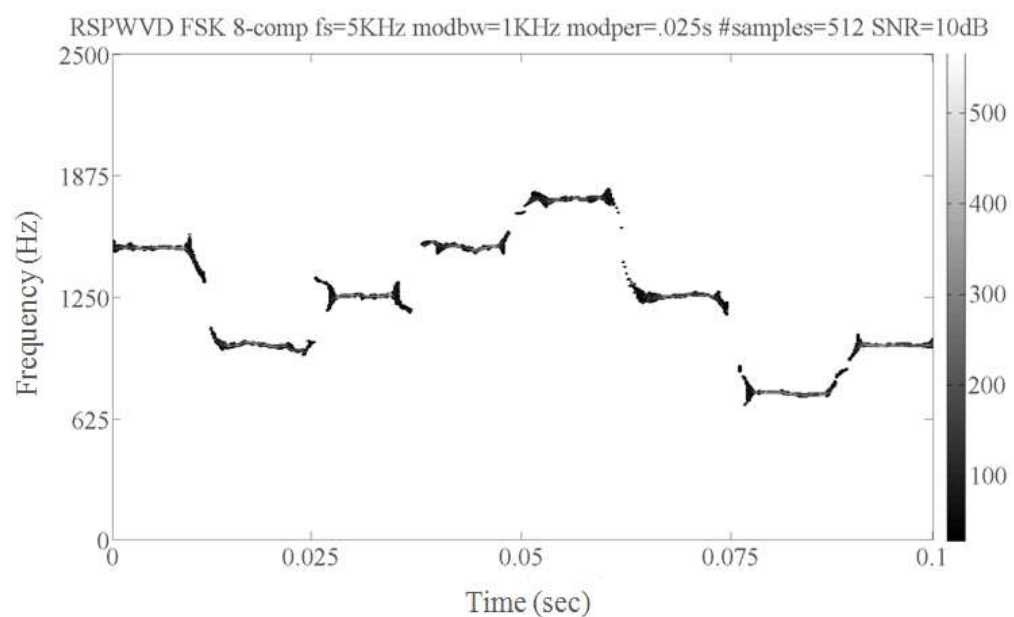

(d)

Fig. 7. Cross-term interference of the WVD ((a) and (c)) and ability of the RSPWVD ((b) and (d)) to reduce cross-term interference. (a) is the WVD and (b) is the RSPWVD for a triangular modulated FMCW signal (512 samples, SNR $=10 \mathrm{~dB})$ and (c) is the WVD and (d) is the RSPWVD for an 8-component FSK signal (512 samples, SNR $=10 \mathrm{~dB}$ ). The WVD displays a lot of cross-term interference. There appears to be one additional triangle signal in the middle of the two-triangle signal in (a) and there appears to be 9 additional signals to go along with the 8-component signal in (c). The RSPWVD has drastically reduced the cross-term interference found in the WVD plots, making for more readable presentations. Notice also that the RSPWVD appears more concentrated than does its WVD counterpart (as per Table 1) 


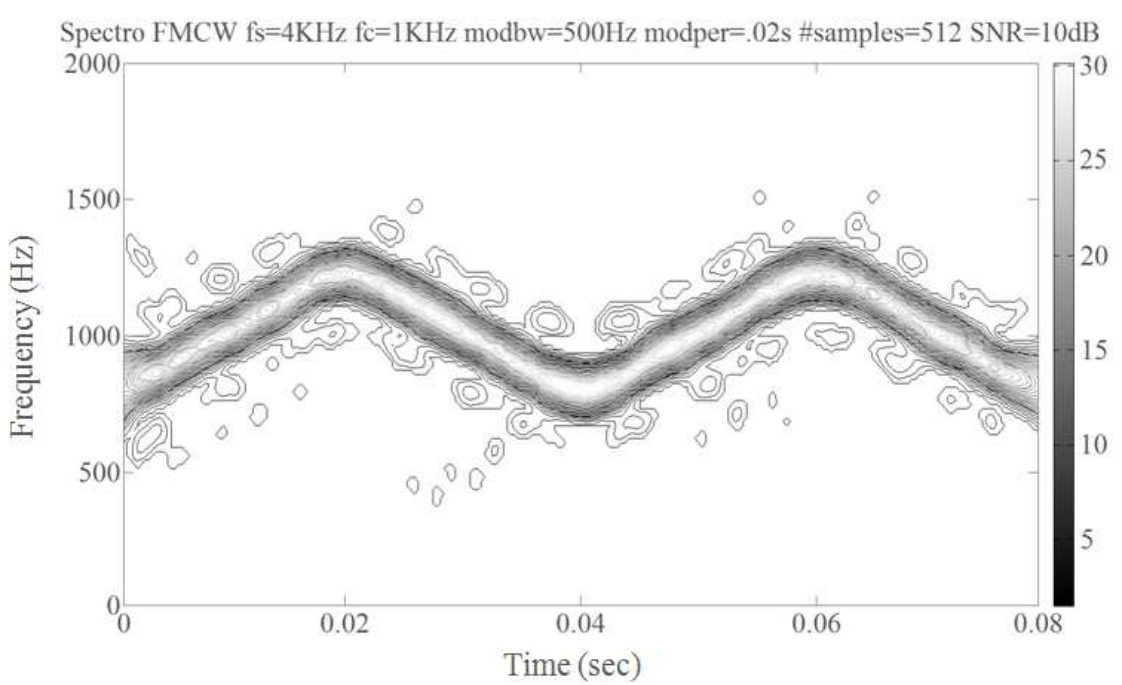

(a)

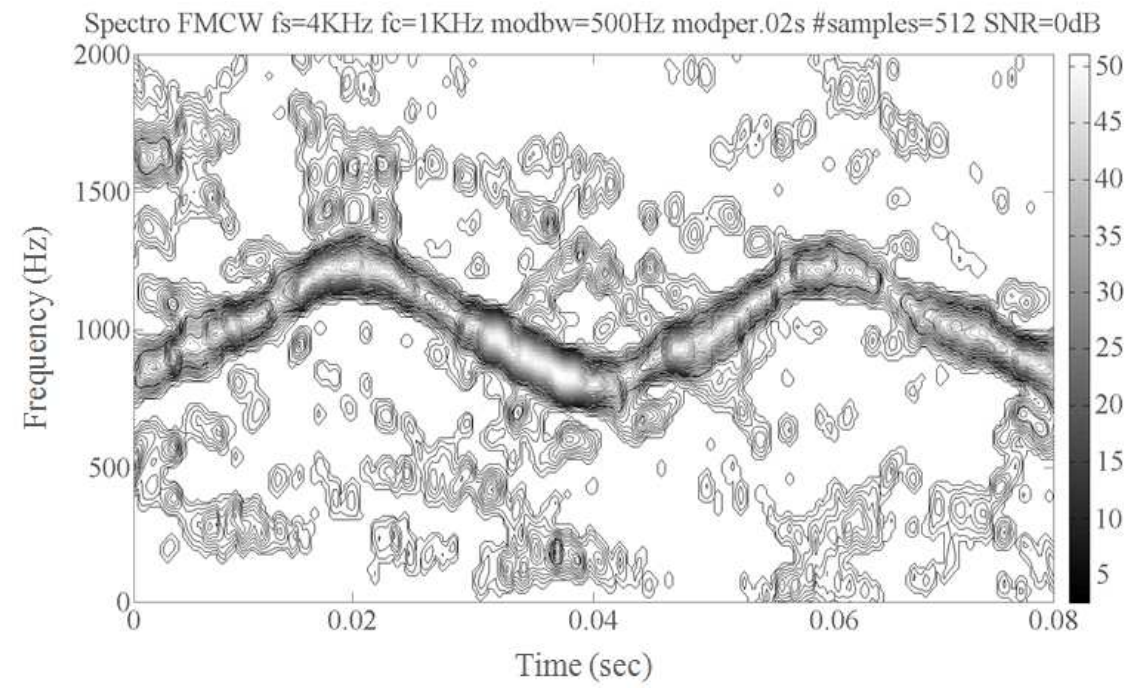

(b)

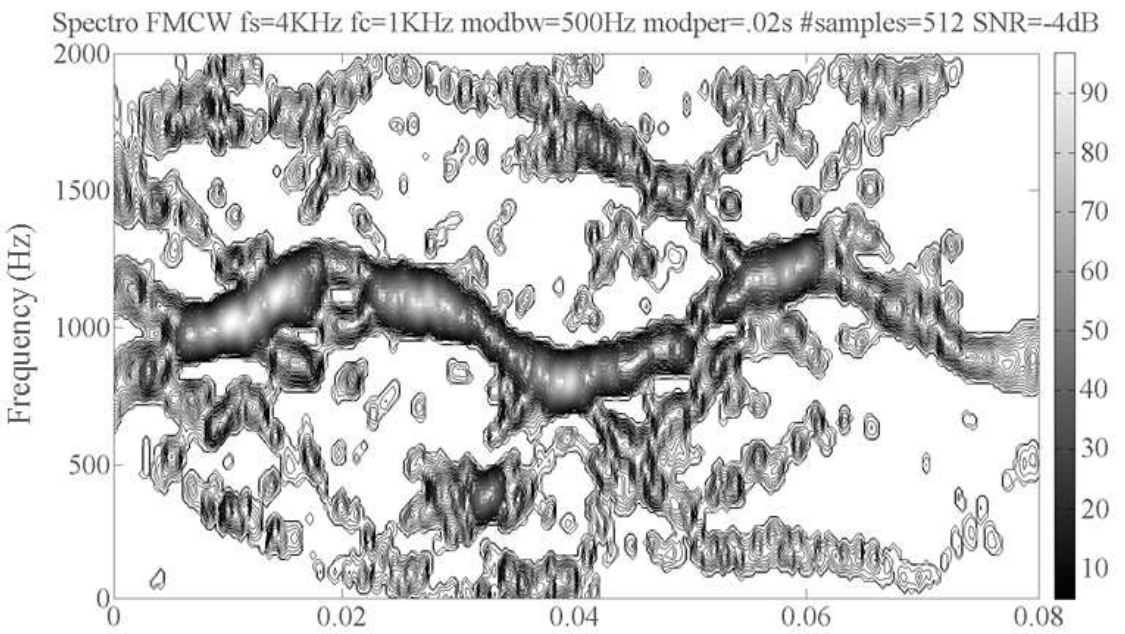

Time (sec)

(c) 


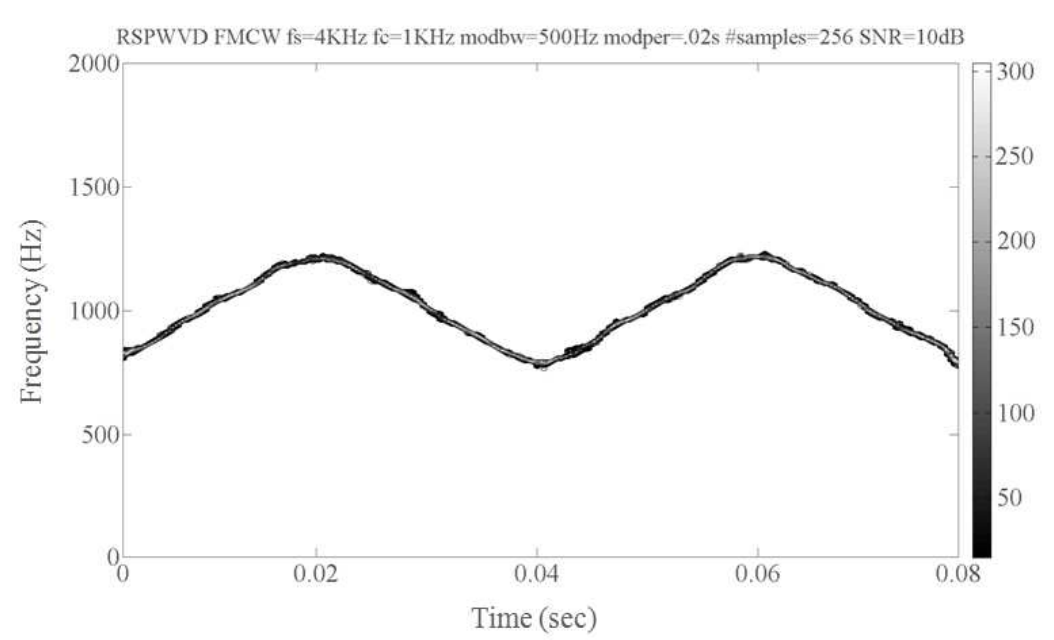

(d)

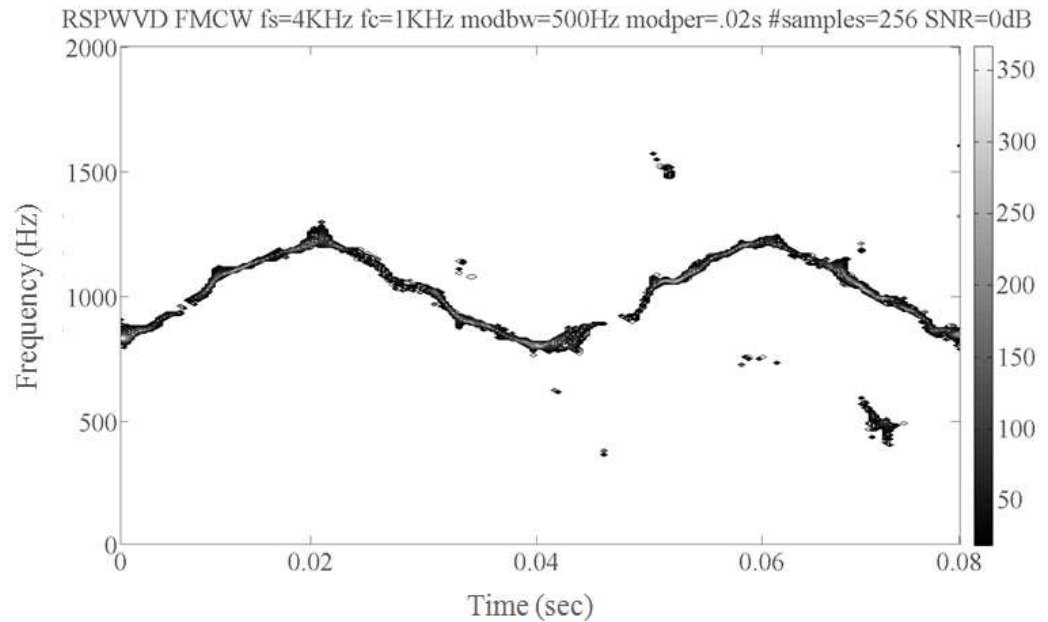

(e)

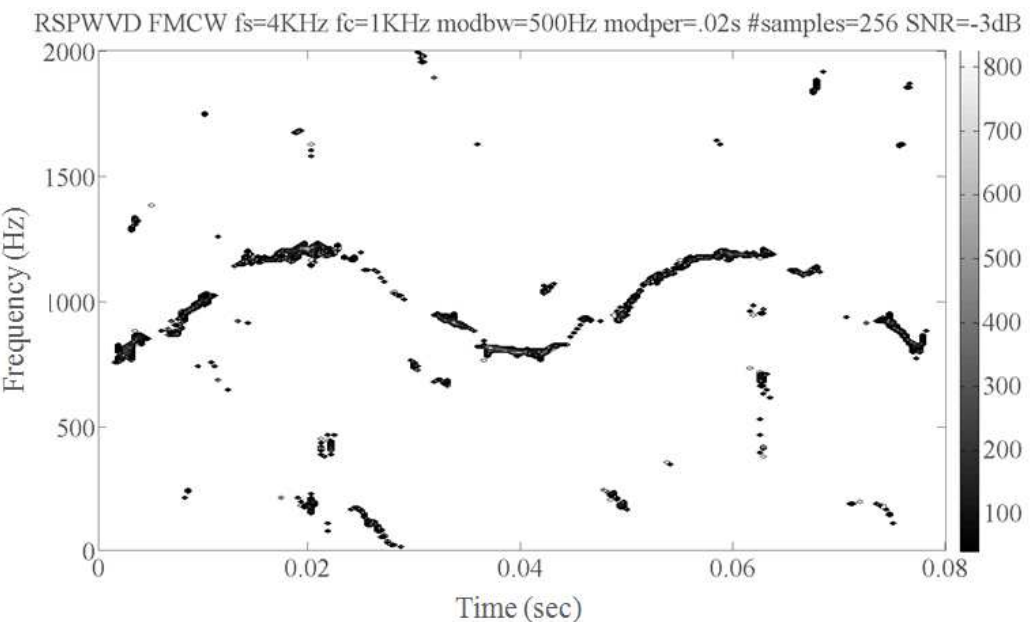

(f)

Fig. 8. Readability degradation due to reduction in SNR. Spectrogram, triangular modulated FMCW, modulation bandwidth $=500$ $\mathrm{Hz}, 512$ samples at (a) $\mathrm{SNR}=10 \mathrm{~dB}$, (b) $\mathrm{SNR}=0 \mathrm{~dB}$, (c) $\mathrm{SNR}=-4 \mathrm{~dB}$. RSPWVD, triangular modulated FMCW, modulation bandwidth $=500 \mathrm{~Hz}, 256$ samples at (d) $\mathrm{SNR}=10 \mathrm{~dB}$, (e) $\mathrm{SNR}=0 \mathrm{~dB}$, (f) SNR $=-3 \mathrm{~dB}$. Readability degrades as SNR decreases, negatively affecting the accuracy of the metrics extracted, as per Table 2 


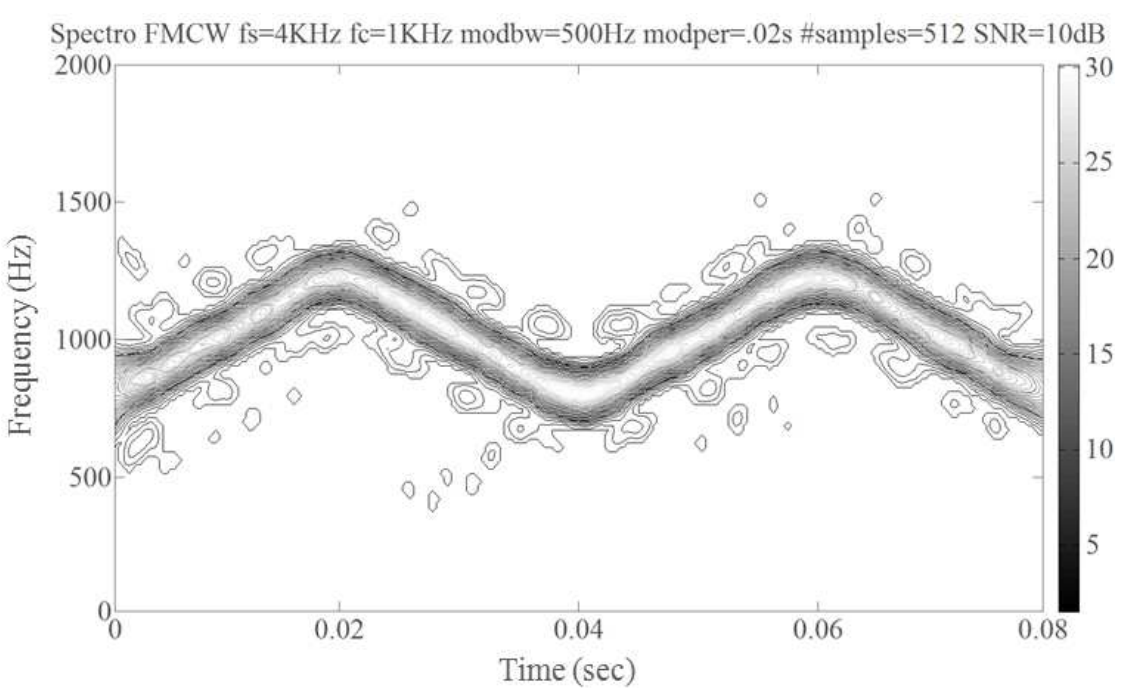

(a)

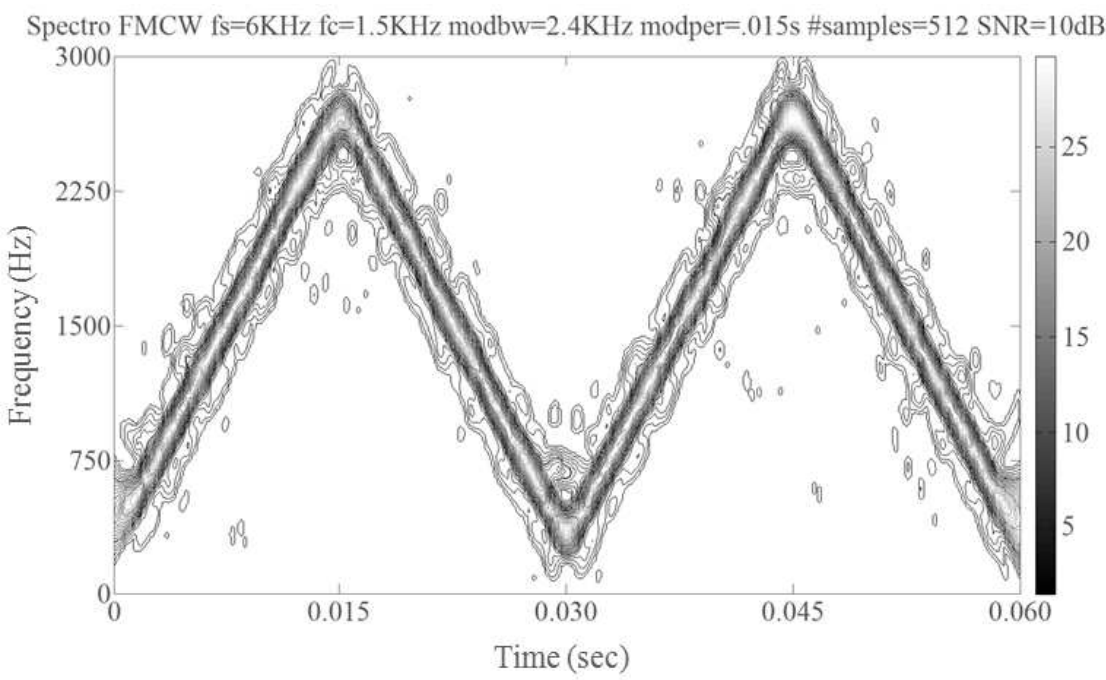

(b)

CWD FMCW fs=4KHz fc $=1 \mathrm{KHz}$ modbw $=500 \mathrm{~Hz}$ modper $=.02 \mathrm{~s}$ \#samples $=512 \mathrm{SNR}=10 \mathrm{~dB}$

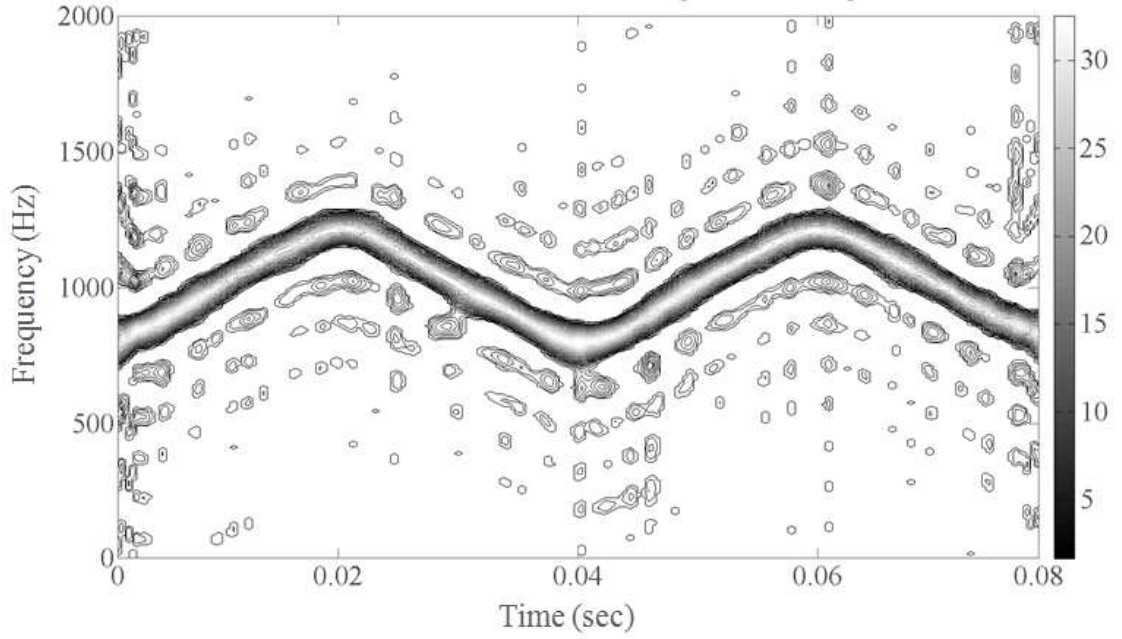

(c) 


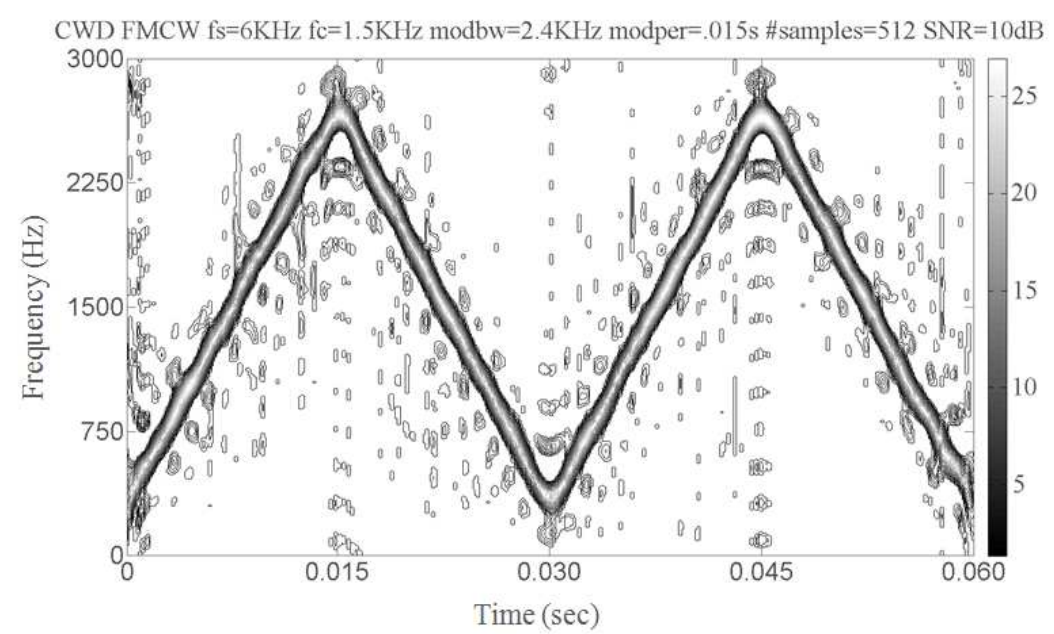

(d)

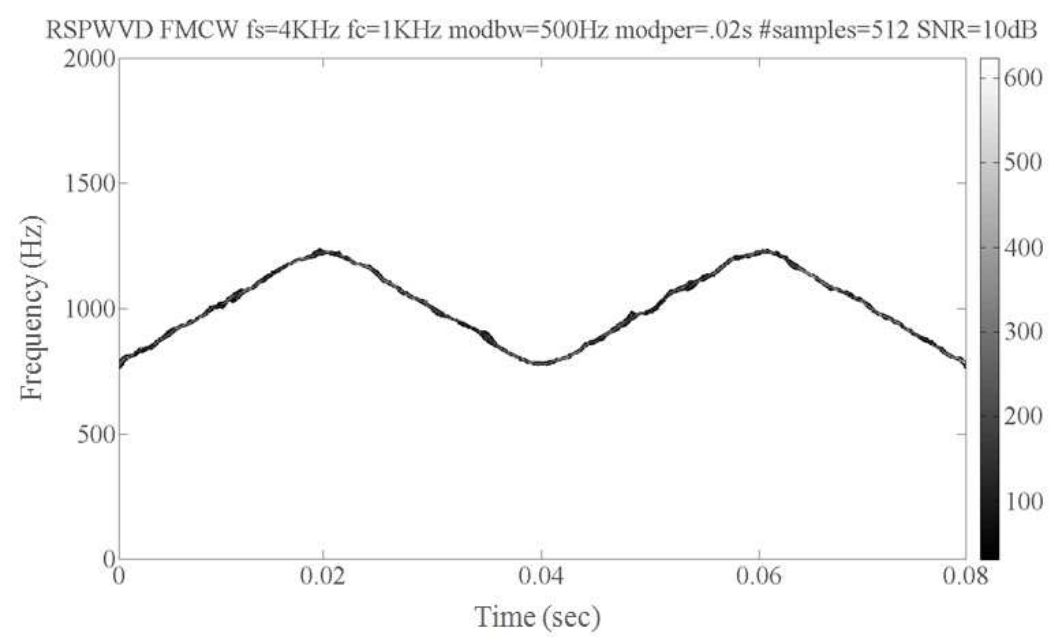

(e)

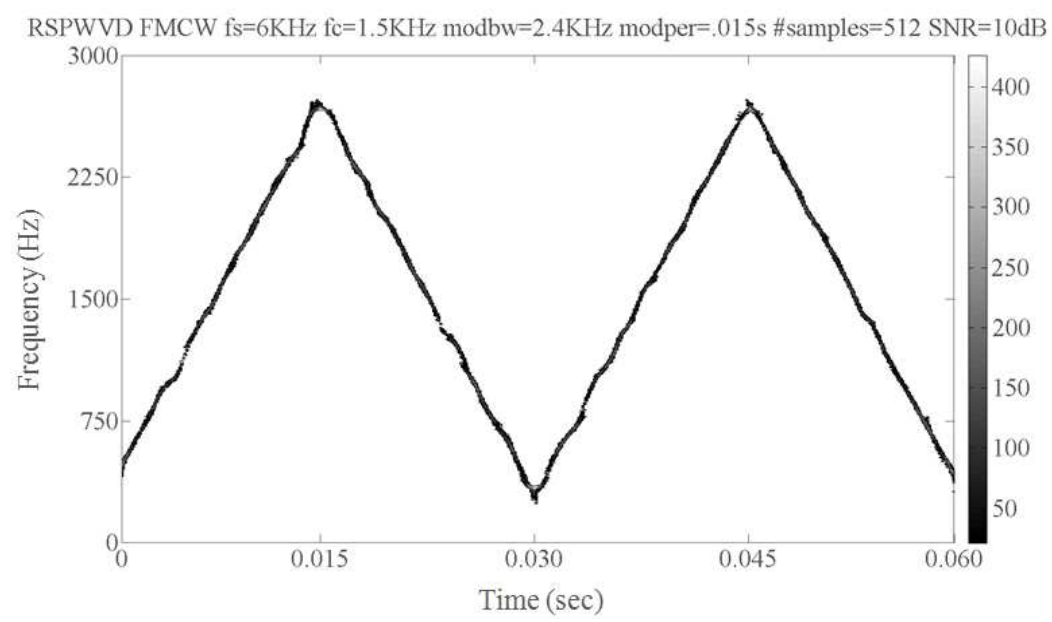

(f)

Fig. 9. Comparison between Task 1 ((a), (c) and (e)) and Task 2 ((b), (d) and (f)). (a) and (b) are both spectrogram plots of a triangular modulated FMCW signal (512 samples, SNR $=10 \mathrm{~dB}$ ), (a) is Task 1 (modulation bandwidth $=500 \mathrm{~Hz})$ and (b) is Task 2 (modulation bandwidth $=2.4 \mathrm{KHz}$ ). (c) and (d) are CWD plots of the same signals and (e) and (f) are RSPWVD plots of the same signals. The Task 2 plots ((b), (d) and (f)) have a larger modulation bandwidth than Task 1 plots ((a), (c) and (e)), therefore the Task 2 signals appear taller and more upright than the Task 1 signals 
From Table 2, it was seen that in general, the percent error of the modulation bandwidth, time-frequency localization ( $\mathrm{x}$ and $\mathrm{y}$ ), percent error of the chirp rate, percent detection and processing time all tended to worsen as the SNR decreased, for both the classical time-frequency analysis techniques and the reassignment method. A visual representation of the degradation of the readability of the time-frequency representation as the SNR decreases is shown in Fig. 8. This confirms an initial hypothesis that as the readability of the timefrequency representation degrades, the accuracy of the metrics degrades as well, as validated in Table 2. It seems plausible that as SNR is lowered, the accuracy of the metrics will decrease, however, it was noted that for the modulation bandwidth, time-frequency localization ( $\mathrm{x}$ and $\mathrm{y}$ ) and chirp rate percent error metrics, the classical time-frequency analysis techniques experienced a greater percent degradation of metrics going from $0 \mathrm{~dB}$ to low SNR (classical TF average low SNR is $-2.58 \mathrm{~dB}$ per Table 1) than from 10 to $0 \mathrm{~dB}(0 \mathrm{~dB}$-low SNR/10-0 dB)): modBW: 22.14/4.19\%; tf-loc x: $6.53 / 4.7 \%$; tf-loc y: $21.39 / 12.32 \%$; cr: $17.44 / 5.68 \%$ ). This highlights the classical time-frequency analysis techniques mediocre performance in a low SNR environment. As noted above, the XFP numbers in Table 2 are representative of the fact that, due to computational complexity, there was no WVD testing accomplished at lower than $10 \mathrm{~dB}$ (except for the 256 sample cases). Had the WVD testing been able to be accomplished at lower than $10 \mathrm{~dB}$ SNR levels for the 512 sample cases, the XFP numbers would have likely increased as the SNR level decreased. Table 2 shows that, by-and-large, the metrics of the reassignment method were more accurate than the metrics of the classical time-frequency analysis techniques at every SNR level.

From Table 3 it was seen that Task 2 (triangular modulated FMCW, modulation bandwidth $=2.4 \mathrm{KHz}$ ) had a much lower percent error for time-frequency localization (y-direction) than Task 1 (triangular modulated FMCW, modulation bandwidth $=500 \mathrm{~Hz}$ ), for both the classical time-frequency analysis techniques ( 2.45 to $6.42 \%)$ and the reassignment method $(0.92$ to $1.94 \%$ ). As per Fig. 9, this is due to the fact that the Task 2 signal is more upright than the Task 1 signal and therefore more of the 'thickness' of the Task 2 signal is in the x-direction than that of the Task 1 signal. It was also noted that percent error of the modulation bandwidth was lower for Task 2 than for Task 1, for both the classical time-frequency analysis techniques (5.90 to $20.97 \%$ ) and the reassignment method (3.36 to $4.59 \%$ ). The modulation bandwidth is a measure from the highest frequency point of a signal to the lowest frequency point of a signal. Therefore, the 'thickness' of a signal will proportionately affect the modulation bandwidth measurement of a 'shorter' signal (Task 1-Fig. 9a, c and e) more than that of a 'taller' signal (Task 2-Fig. 9b, d and $\mathrm{f}$ ). Because of this, the modulation bandwidth percent error will be lower for Task 2 (the 'taller' signal) than for Task 1 (the 'shorter' signal). It should be noted that though the definition of modulation bandwidth is the measure in frequency from the highest frequency value (at a $20 \%$ threshold of the maximum intensity for this testing) of a signal to the lowest frequency value (at a $20 \%$ threshold of the maximum intensity) of a signal, an experienced intercept receiver signal analyst may choose to manually 'override' the $20 \%$ threshold value and manually measure the modulation bandwidth of a 'thick' signal at points which he believes would give a more accurate modulation bandwidth measurement. This method may work for time-frequency representations with good readability, but becomes more difficult to accomplish as the signal readability degrades due to low SNR and cross-term interference and the more so for a less experienced intercept receiver signal analyst. Table 3 also shows that the chirp rate percent error is lower for Task 2 than for Task 1 for both the classical timefrequency analysis techniques (5.77 to $12.09 \%$ ) and the reassignment method ( 2.86 to $4.89 \%$ ). This follows from the fact that the modulation bandwidth percent error is lower for Task 2 than Task 1 and that chirp rate, which equals modulation bandwidth/modulation period, is directly proportional to the modulation bandwidth.

From Table 3 it is also noted that the carrier frequency percent error for the FSK waveforms (Task 3 and Task 4) is lower than the carrier frequency percent error for the triangular modulated FMCW waveforms (Task 1 and Task 2) for both the classical time-frequency analysis techniques (Task $3=0.65 \%$; Task $4=0.40 \%$; Task $1=4.02 \%$; Task $2=8.15 \%$ ) and the reassignment method (Task $3=0.45 \%$; Task $4=0.37 \%$; Task $1=$ $4.15 \%$; Task $2=4.48 \%$ ). This is due to the fact that the FSK waveforms are basically frequency tones, with each frequency tone representing a different carrier frequency. Also noted was the fact that the modulation period percent error for the FSK waveforms (Task 3 and Task 4) was greater than the modulation period percent error for the triangular modulated FMCW waveforms (Task 1 and Task 2), for both the classical time-frequency analysis techniques (Task $3=10.7 \%$; Task $4=17.47 \%$; Task $1=0.70 \%$; Task $2=0.41 \%$ ) and the reassignment method (Task $3=6.25 \%$; Task $4=12.0 \%$; Task $1=$ $0.78 \%$; Task $2=0.56 \%$ ). This is due to the fact that for a triangular modulated FMCW signal, the modulation period is the measure of the point where the first and second chirp legs of the signal meet to the point where the second and third chirp legs of the signal meet (measured in the x-direction) and the location of these two points changes very little based on time-frequency localization (signal 'thickness') or the SNR level (Fig. 6a 
and $b$ ). For the FSK waveform, the modulation period is a measure of the width of the entire signal component (measured in the x-direction) and can vary drastically based on time-frequency localization and SNR level (Fig. 6c and d). From Table 3, it was also noted that the modulation period percent error for the 4-component FSK signal (Task 3) was smaller than the modulation period percent error for the 8-component FSK signal (Task 4) (for classical TF: Task $3=10.7 \%$; Task $4=$ $17.47 \%$; for RM: Task $3=6.25 \%$; Task $4=12.0 \%$ ). This may be due to the fact that the size of the gap between signal components in the $\mathrm{x}$-direction for the 4component signal is roughly the same as the size of the gap between signal components for the 8-component signal, leaving less signal space in the $\mathrm{x}$-direction for the 8-component signal (i.e., shorter components in the $\mathrm{x}$ direction) and therefore a larger percent error of the modulation bandwidth (measure of the $\mathrm{x}$-direction width of the signal). Another observation from Table 3 was that the low SNR value for the 4-component FSK signal (Task 3) was about $1 \mathrm{~dB}$ lower than the 8-component FSK signal (Task 4) (for classical TF: Task $3=-2.67 \mathrm{~dB}$; Task $4=-1.67 \mathrm{~dB}$; for RM: Task $3=-3.33 \mathrm{~dB}$; Task $4=$ $2.5 \mathrm{~dB}$ ). This may be due to the fact that the Task 3 signal has only 4 components, each of which is about twice as long as the 8-component signals of Task 4. This means that at low SNR levels, the Task 3 signal has a better chance of at least a portion of each of its 4 (longer) signal components exceeding the low SNR threshold than does the Task 4 signal with its 8 (shorter) components.

Recapping, the metrics data backs up the following introductory assumptions:

- The classical time-frequency analysis techniques are deficient in the areas of time-frequency localization and cross-term interference, making for poor readability and consequently, for less accurate detection and parameter metrics extraction of LPI radar signals

- Since the reassignment method is, in theory, a perfectly localized distribution for chirps, tones and impulses, it should (and does) work well with LPI radar signals such as the triangular modulated FMCW and the FSK

- The smoothing and squeezing qualities of the reassignment method make for better readability and consequently more accurate signal detection and parameter metrics extraction of LPI radar signals, making for a potentially more informed and effective intercept receiver environment

\section{Conclusion}

Though time-frequency analysis techniques, such as the WVD, CWD, spectrogram and scalogram, are quickly replacing Fourier-based analysis for digital intercept receivers, it was shown through testing plots and results metrics that these time-frequency analysis techniques suffer from a lack of readability due to deficiencies of poor time-frequency localization and cross-term interference. It was observed that this lack of readability led to inaccurate detection and parameter extraction metrics of the LPI radar signals.

The reassignment method was introduced as being a potential solution to these deficiencies. Because of its theoretical perfectly localized distribution for chirps, tones and impulses, the reassignment method worked well for the triangular modulated FMCW and the FSK LPI radar waveforms used for this testing.

Simulations were presented that compared timefrequency representations of the classical time-frequency techniques with those of the reassignment method. Based on a number of metrics, experimental results demonstrated that the 'squeezing' and 'smoothing' qualities of the reassignment method did indeed lead to improved readability over the classical time-frequency analysis techniques and consequently, provided more accurate signal detection and parameter extraction metrics (smaller percent error from true value) than the classical time-frequency analysis techniques.

In summary, this study provides evidence that the reassignment method has the potential to outperform the classical time-frequency analysis techniques, which are the current state-of-the-art, cutting-edge techniques for this arena. An improvement in performance could easily translate into saved equipment and lives.

Future plans include automation of the metrics extraction process, analysis of additional low probability of intercept radar waveforms of interest and analysis of other real-world low probability of intercept radar signals utilizing more powerful computing platforms.

\section{Acknowledgment}

The researchers gratefully acknowledge the technical support provided by Dr. Andrew J. Noga of the Air Force Research Laboratory.

\section{Funding Information}

This research was internally supported.

\section{Author's Contributions}

D. Stevens: Developed the mathematical modeling, participated in collecting and analyzing data, contributed to the writing of the manuscript.

S. Schuckers: Participated in analyzing the data, organized the manuscript, contributed to the writing of the manuscript. 


\section{Ethics}

A preliminary version of this paper was presented at the 2nd Annual AOC Symposium on Low Probability of Intercept RADAR and Counter-LPI Technology, Feb. 2011. Another preliminary version of this paper was presented at MILCOM 2014, Oct. 2014.

\section{References}

Anjaneyulu, L., N. Murthy and N. Sarma, 2009. Identification of LPI radar signal modulation using bi-coherence analysis and artificial neural networks techniques.

Auger, F. and P. Flandrin, 1994. The why and how of time-frequency reassignment. Proceedings of the IEEE-SP International Symposium on TimeFrequency and Time-Scale Analysis, Oct. 25-28, IEEE Xplore Press, Philadelphia, PA., pp: 197-200. DOI: 10.1109 /TFSA.1994.467259

Auger, F. and P. Flandrin, 1995. Improving the readability of time-frequency and time-scale representations by the reassignment method. IEEE Trans. Signal Process., 43: 1068-1089. DOI: $10.1109 / 78.382394$

Auger, F., P. Flandrin, P. Goncalves and O. Lemoine, 1996. Time-frequency toolbox users manual. Centre National de la Recherche Scientifique and Rice University.

Boashash, B., 2003. Time Frequency Signal Analysis and Processing: A Comprehensive Reference. 1st Edn., Elsevier, Oxford, England, ISBN-10: 0080443354, pp: 743 .

Choi, H.I. and W.J. Williams, 1989. Improved timefrequency representation of multicomponent signals using exponential kernels. IEEE Trans. Acoust. Speech Signal Process., 37: 862-871. DOI: $10.1109 /$ ASSP.1989.28057

Cirillo, L., A. Zoubir and M. Amin, 2008. Parameter estimation for locally linear FM signals using a time-frequency Hough transform. IEEE Trans. Signal Process., 56: 4162-4175.

DOI: 10.1109/TSP.2008.924797

Cohen, L., 2002. The Wavelet Transform and TimeFrequency Analysis. In: Wavelet Transforms and Their Applications, Debnath, L. (Ed.), Springer Science and Business Media, Boston, ISBN-10: 0817642048, pp: 3-22.

Cohen, L., 1995. Time-Frequency Analysis. 1st Edn., Prentice Hall, Englewood, ISBN-10: 0135945321, pp: 299.

De Luigi, C. and E. Moreau, 2002. Wigner-Ville and polynomial Wigner-Ville transforms in the estimation of nonlinear FM signal parameters. Proceedings of the IEEE International Conference on Acoustics, Speech and Signal Processing, May 13-17, IEEE Xplore Press, Orlando, Fl., pp: 1433-1436. DOI: 10.1109/ICASSP.2002.5745822
Faulconbridge, I., 2002. Radar Fundamentals. 1st Edn., Argos Press, Canberra, ISBN-10: 0958023816, pp: 267.

Flandrin, P., F. Auger and E. Chassande-Mottin, 2003. Time-Frequency Reassignment: From Principles to Algorithms. In: Applications in Time-Frequency Signal Processing, Papandreou-Suppappola, A. (Ed.), CRC Press, ISBN-10: 1420042467, pp: 179-203.

Galleani, L., L. Cohen and A. Noga, 2006. A timefrequency approach to the adjustable bandwidth concept. Digital Signal Process., 16: 454-467. DOI: $10.1016 /$ j.dsp.2005.08.001

Gau, J.Y., 2002. Analysis of Low Probability of Intercept (LPI) Radar Signals Using the Wigner Distribution. 1st Edn., ISBN-10: 1423507584, pp: 166.

Grishin, Y. and D. Janczak, 2007. Computer-aided methods of the LPI radar signal detection and classification. Proceedings of the SPIE Photonics Applications in Astronomy, Communications, Industry and High-Energy Physics, (HEP' 07), SPIE, pp: 1-5. DOI: 10.1117/12.784898

Guanghua, C., M. Shiwei, Q. Tinghao, W. Jian and C. Jialin, 2008. Wigner-Ville distribution and cross Wigner-Ville distribution of noisy signals. J. Syst. Eng. Electron., 19: 1053-1057. DOI: $10.1016 / \mathrm{S} 1004-4132(08) 60196-6$

Gulum, T., 2007. Autonomous non-linear classification of LPI radar signal modulations. MSc Thesis, Naval Postgraduate School, Monterey.

Hippenstiel, R., M. Fargues, I. Moraitakis and C. Williams, 2000. Detection and parameter estimation of chirped radar signals. Naval Postgraduate School, Monterey.

Hlawatsch, F. and G.F. Boudreaux-Bartels, 1992. Linear and quadratic time-frequency signal representations. IEEE Signal Process. Mag., 9: 21-67. DOI: 10.1109/79.127284

Lari, F. and A. Zakhor, 1992. Automatic classification of active sonar data using time-frequency transforms. Proceedings of the IEEE-SP International Symposium on Time-Frequency and Time-Scale Analysis, Oct. 4-6, IEEE Xplore Press, Victoria, BC., pp: 21-24. DOI: 10.1109/TFTSA.1992.274242

Li, X. and G. Bi, 2008. A new reassigned time-frequency representation. Proceedings of the 16th European Signal Processing Conference, Aug. 25-29, Lausanne, Switzerland, pp: 1-4.

Liang, Y., L. Zhang, M. Xing and Z. Bao, 2009. Highspeed ground moving target detection research using triangular modulation FMCW. Front. Electr. Electron. Eng., China, 4: 127-133. DOI: $10.1007 / \mathrm{s} 11460-009-0032-\mathrm{z}$

Milne, P.R. and P.E. Pace, 2002. Wigner distribution detection and analysis of FMCW and P-4 polyphase LPI waveforms. Proceedings of the IEEE International Conference on Acoustics, Speech and Signal Processing, May 13-17, IEEE Xplore Press, Orlando, Fl., pp: 3944-3947. DOI: 10.1109/ICASSP.2002.5745520 
Mitra, S.K., 2001. Digital Signal Processing: A Computer-Based Approach. 2nd Edn., McGrawHill, Boston, MA., ISBN-10: 0072321059, pp: 896.

Pace, P.E., 2009. Detecting and Classifying Low Probability of Intercept Radar. 1st Edn., Artech House, Boston, ISBN-10: 159693235X, pp: 857.

Stephens, J., 1996. Advances in signal processing technology for electronic warfare. IEEE Aerospace Electronic Syst. Magaz., 11: 31-38. DOI: $10.1109 / 62.544024$

Upperman, T., 2008. ELINT signal processing using Choi-Williams distribution on reconfigurable computers for detection and classification of LPI emitters. MSc Thesis, Naval Postrgraduate School.

Wiley, R.G., 2006. ELINT: The Interception and Analysis of Radar Signals. 1st Edn., Artech House, Boston, ISBN-10: 1580539254, pp: 451.
Williams, W. and J. Jeong, 1992. Reduced Interference Time-Frequency Distributions. In: Time-Frequency Signal Analysis: Methods and Applications, Boashash, B. (Ed.), Longman-Cheshire/Wiley, Melbourne/N.Y.

Wong, K., T. Davidson and S. Abelkader, 2009. Detection of low probability of intercept radar signals. Defence R\&D Canada-Ottawa Contract Report.

Xie, C., K. Zhang, K. Feng, C. Guo and J. Xu, 2008. Target detecting based on improved WVD-HT for radio frequency interference to UWB-SAR. Proceedings of the 1st International Conference on Intelligent Networks and Intelligent Systems, Nov. 1-3, IEEE Xplore Press, Wuhan, pp: 604-607. DOI: $10.1109 /$ ICINIS.2008.173 